\title{
Von der Zootomie zur neuzeitlichen Pferdeheilkunde - Entwicklung der Tiermedizin in Berlin-Mitte
}

Rudolf K. H. Wernicke

\section{Einleitung}

Von der Öffentlichkeit kaum bemerkt, steht mitten in Berlin im ehemaligen Tierarzneischulgarten ein denkmalsgeschütztes Gebäude: Die "Alte Zootomie". Neuerdings haben Denkmalsschützer und die Mitglieder der "Gesellschaft historisches Berlin e. V." sowie Einzelvertreter der Berliner veterinärmedizinischen Lehr- und Forschungsstätten und nun endlich auch die Bundestierärztekammer die Notwendigkeit erkannt, dieses vom Verfall bedrohte kulturhistorisch wertvollen Gebäude für die Nachwelt zu erhalten (Abbildung 1).

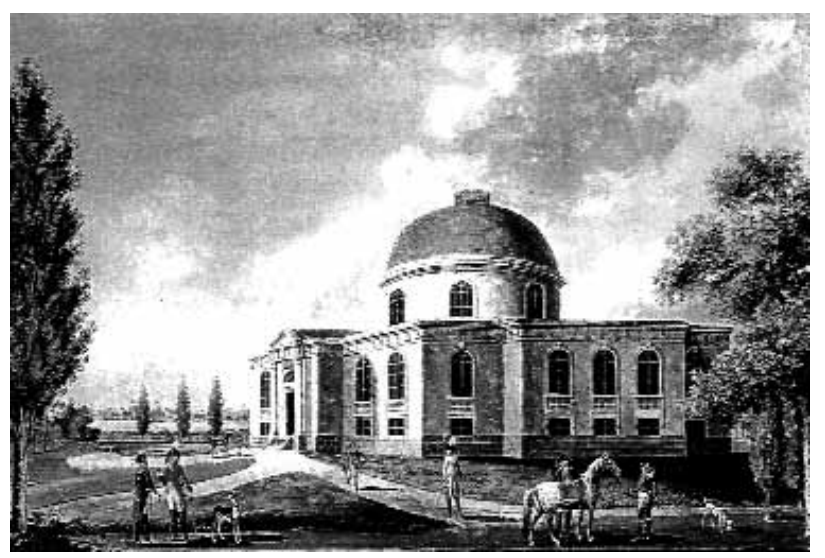

Abb 4 Die Königliche Tierarzneischule mit dem Schimmelwallach "Condé", dem Leibreitpferd Friedrich II, im Vordergrund. Nach einem Gemälde des Kunstmalers Ruff

Leider wurden in einigen Veröffentlichungen über die "Alte Zootomie" auch Fakten zusammengetragen, welche die Geschichte des Bauwerkes und seiner Nutzung nicht immer korrekt wiedergeben. Nachfolgend wird daher der Versuch unternommen, über die Geschichte des Gebäudes und die damit zusammenhängende Entwicklung der Pferdeheilkunde an der Berliner tierärztlichen Lehr- und Forschungsstätte einen kurzen Abriss vorzustellen. Grundlage dieser Darstellung bilden die Festschriften zum 50. (Albers 1841), 100. (Schütz 1890), 150. (Beutner 1940) und 200. (Hass 1989 und Deutrich 1990) Jubiläum der Berliner Tierarzneischule (1790-1887), Tierärztlichen Hochschule (1887-1934) und Veterinärmedizinischen Fakultät (1937-1990) sowie andere veterinärhistorische, medizinhistorische und sonstige Veröffentlichungen, die für dieses Thema von Interesse sind.

\section{Am Anfang standen zwei Jahrzehnte Empirie und hand- werkliche Ausbildung (1790-1817)}

Zum Gründungsmythos der neuzeitlichen Veterinär- bzw. Tierarzneischulen, die im 18. Jahrhundert in Europa entstanden, gehörte das Argument, sie seien zur Ausbildung von Spezialisten für die Tierseuchenbekämpfung eingerichtet worden (Schwarz 1967). Nach der Errichtung der ersten Veterinärschulen in Lyon und in Alfort bei Paris sowie der "Pferdekurund Operationsschule" in Wien (vgl. Tabelle 1) stand dieses Argument zwar Ende der 60er Jahre das 18. Jahrhunderts im Vordergrund, als der Leibarzt von Friedrich II., dem Großen $\left({ }^{*} 1712\right.$, seit 1740 König, +1786) Christian Andreas Cot henius (1708-1789), gestützt auf seine Erfahrungen als Kreisphysikus der Prignitz (1740-1747), einen ersten Entwurf für die Errichtung einer "Vieharzneischule" vorgelegt hatte. An dieser Schule, für die auch ein Bauplan vorlag und ein Standort vor dem Brandenburger Tor im Tiergarten an der Spree in Aussicht genommen war, wollte Cothenius Physiker (Amtsärzte) für die Tierseuchenbekämpfung und Viehärzte für die Landwirtschaft ausbilden lassen (Rieck 1928). Doch dann lehnte der "Alte Fritz" den Vorschlag zur Errichtung einer "École vétérinaire" ab, da dafür kein "convenabler Fonds" vorhanden sei (Koch 1965, Deutrich 1990) und das Projekt wurde auf Eis gelegt.

Nach französischem Vorbild wurden nun aber ab 1769 auch in Italien, Dänemark, Schweden, Sachsen, Hannover und Baden Veterinärschulen gegründet (Schmaltz 1936). Bei diesen Schulen stand vorerst die Pferdeheilkunst im Vordergrund. Dafür gibt es offenbar mehrere Gründe. In der veterinärhistorischen Literatur wurde bisher kaum zur Kenntnis genommen, dass sich in der Mitte des 18. Jahrhunderts, der Waren- und Personenverkehr mehr und mehr von den Wasserstrassen auf den Landweg verlagerte. Mit den von den europäischen Staaten errichteten Postmonopolen entstand jetzt auch auf dem Landwege ein Verkehrsnetz, auf dem für den Postgut- und Personenverkehr Reit- und Fahrposten im Einsatz waren (Wernicke 1996). Schließlich erlangten im 18. Jahrhundert auch die Kavallerie und die Artillerie als kriegsentscheidende Waffengattungen eine zunehmende Bedeutung. Diese Entwicklungen in Friedens- und Kriegszeiten erforderten einen erhöhten Bedarf an geeigneten Pferden. Unverkennbar besteht hier auch ein Zusammenhang mit der im 18. Jahrhundert beginnenden staatliche Verwaltung der bisherigen Hof- und landesherrlichen Gestüte (Stang und Wirth 
1927), die auch den Einsatz von Rossärzten verlangte (vgl. Tabelle 1).

Dies alles waren Gründe genug, die Ausbildung an den Veterinär- bzw. Tierarzneischulen auf die Pferdeheilkunde mit Einschluss der Hufbeschlagskunde auszurichten. Nach dem Tode des "Alten Fritzen" wurde der damalige Kammerherr in der sächsischen Gestütsverwaltung, der Graf Karl Heinrich August von Lindenau (1755-1842) vom Preussenkönig Frie drich Wilhelm II. (*1744, seit 1786 König, +1797) zum Major befördert und zum Reisestall-

Tab 1 Gestütsgründungen und Errichtung der ersten europäischen Veterinär- bzw. Tierarzneischulen (zusammengestellt nach Pusch 1909, Stang und Wirth 1927, Rieck 1934, Schmaltz 1936, Rieck 1934, 1937, von den Driesch und Peters 2003).

\begin{tabular}{|c|c|c|c|}
\hline \multicolumn{2}{|c|}{ Gestüte } & \multicolumn{2}{|c|}{ Veterinär- bzw. Tierarzneischulen } \\
\hline Jahr & Standort & Jahr & Standort \\
\hline \multicolumn{4}{|l|}{ Frankreich } \\
\hline 1573 & Mömpelgard/Montbéliard & 1762 & Lyon \\
\hline 1714 & La Pin & 1764 & Alfort \\
\hline 1748 & Strassburg im Elsass & & \\
\hline 1751 & \begin{tabular}{|l|l|} 
Pompadeur \\
\end{tabular} & & \\
\hline \multicolumn{4}{|c|}{ Ósterreich - Ungarn } \\
\hline 1562 & Kladruby bei Pardubice & 1767 & Wien, Pferdecur- u. Op.Schule \\
\hline 1580 & Lippizza im Karst & 1777 & Wien, k. k. Tierspital \\
\hline Um 1700 & Liechtenstein, Dietrichsstein und Schwarzenberg & & \\
\hline $1770 / 80$ & Salzburg & & \\
\hline 1785 & Mezö - Hegyes & & \\
\hline 1792 & Radauz in der Bukowina & & \\
\hline 1798 & Piber bei Graz & & \\
\hline \multicolumn{4}{|l|}{ Italien } \\
\hline 1639 & San Rossore bei Pisa & 1769 & Turin \\
\hline \multicolumn{4}{|l|}{ Dänemark } \\
\hline 1562 & Frederiksborg & 1773 & Kopenhagen \\
\hline \multicolumn{4}{|l|}{ Schweden } \\
\hline 1658 & Flyinge & 1774 & Skara \\
\hline \multicolumn{4}{|l|}{ Sachsen } \\
\hline 1588 & Kalkreuth & 1774 & Dresden \\
\hline 1590 & Torgau & & \\
\hline 1666 & Repitz bei Torgau & & \\
\hline 1694 & Paudritzsch & & \\
\hline 1722 & Graditz & & \\
\hline 1733 & Moritzburg & & \\
\hline 1757 & In allen kursächsischen Gestüten sind Rossärrte tätig & & \\
\hline 1767 & $\begin{array}{l}\text { Neben den Hofgestituen entstehen in Sachsen } \\
\text { Landesbeschälstationen }\end{array}$ & & \\
\hline \multicolumn{4}{|c|}{ 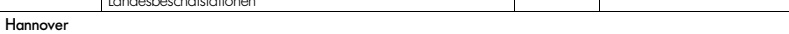 } \\
\hline 1735 & Celle & 1778 & Hannover \\
\hline \multicolumn{4}{|l|}{ Baden } \\
\hline 1753 & Durlach & 1784 & Karlsuhe \\
\hline Brandenburg & Preussen & & \\
\hline Vor & Vorwerk - Plawen (Amt Falkenburgk) sowie Stutenhöfe in Küstrin & 1790 & Berlin \\
\hline$\frac{1600}{1600}$ & $\begin{array}{l}\text { und Wollup (Amt Lebus) } \\
\text { Driesen mit Fohlengarten }\end{array}$ & & \\
\hline 17. Jhdt. & $\begin{array}{l}\text { Mehrere kleinere märkische Stutereien, darunter auch } \\
\text { Neustadt/Dosse }\end{array}$ & & \\
\hline 1713 & Kgl. AO zur Errichtung von Landgestititen & & \\
\hline 1717 & Stuterei Ragnit/Opr. & & \\
\hline 1732 & Trakehnen & & \\
\hline 1749 & Stutereie in Georgenburg/Opr. & & \\
\hline 1768 & Maultiergestüt Neustadt/Dosse & & \\
\hline $1770 / 80$ & Insterburg/Opr. & & \\
\hline 1787 & $\begin{array}{l}\text { Hauptgestüte in Neustadt/ Dosse und Trakehnen sowie } \\
\text { Landgestute Georgenburg und Oletzo/Opr. }\end{array}$ & & \\
\hline 1788 & Landgestüt in Marienwerder/Npr. & & \\
\hline 1789 & Landgestüte in Neustadt/Dosse und Lieberwalde/Kumark & & \\
\hline \multicolumn{4}{|c|}{ J } \\
\hline 1571 & Rohrenfeld & 1790 & München \\
\hline 1749 & Bamberg & & \\
\hline 1754 & $\begin{array}{l}\text { Schleissheim bei München: Beginn des staatlichen } \\
\text { Gestüswesesens in Bayern }\end{array}$ & & \\
\hline 1769 & Ansbach und Zweibrücken in der Kunfalz & & \\
\hline 1793 & Klingenhof in Mittelfranken & & \\
\hline
\end{tabular}

meister ernannt. Er wurde Ende 1786 auch mit der "Leitung der gesamten Details des (preussischen) Obermarstalldepartements" (Lutz 2003, Wernicke 2004, Mielke 2005) betraut. In dieser Funktion veranlasste er 1787 seinen König zum Erlass einer Kabinettsorder, nach der sich die preussische Armee im Inland remontieren sollte, was zuvor unter Friedrich II. nicht der Fall war. Zugleich wurde vom Grafen ein 1. Preussisches Landgestütsreglement erlassen, das die Reorganisation des preussischen Gestütswesens vorsah: Die Gestüte in Trakehnen und Neustadt/Dosse wurden Hauptgestüte. In Neustadt/Dosse und in Lieben- walde sowie in Ost- und Westpreussen wurden Landgestüte eingerichtet.

Im gleichen Jahr wurde offenbar auch wieder auf Veranlassung von dem inzwischen zum Vize - Oberstallmeister ernannten Grafen von Lindenau vom König bestimmt, dass in Berlin eine "École vétérinaire" errichtet werden soll, "... weil der Schade, der aus Mangel an guten Ross- und Viehärzten entstanden, für das Land und die Cavallerie von den allertraurigsten Folgen sey" (Albers 1841, Schütz 1890, Koch 1965, Deutrich 1990).

Jetzt wurde, wie das auch bei den bereits existierenden Tierarzneischulen mit Ausnahme von Hannover der Fall gewesen war, ein für ein Lehramt vorgesehener Kandidat an eine fran-

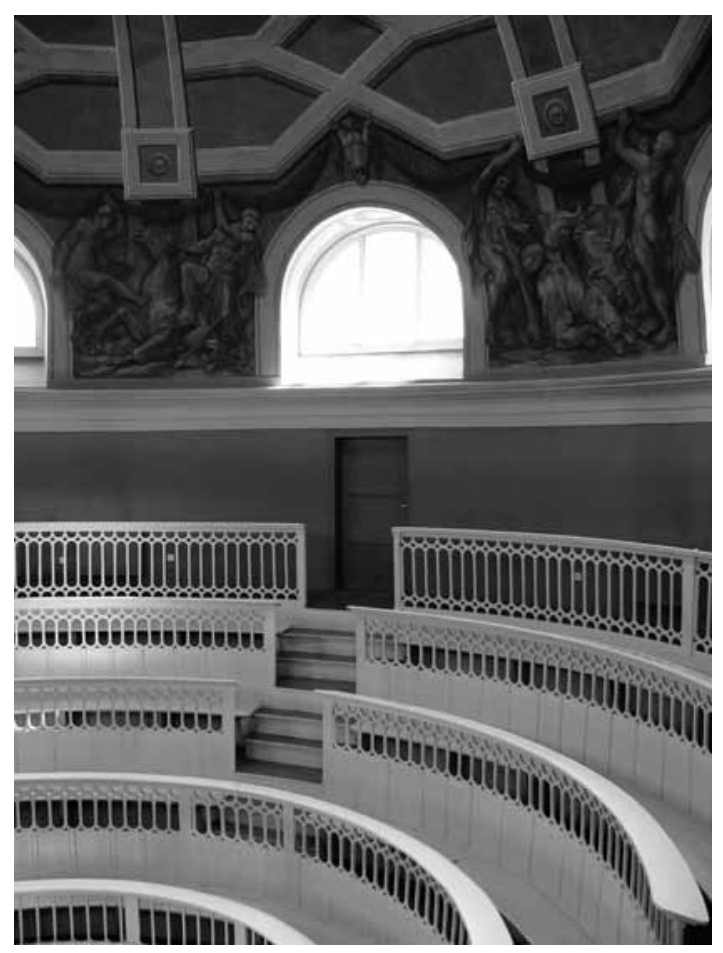

Abb 2 Innenansicht des zentralen Hörsaals

zösische Veterinärschule geschickt, um dort in die Tierarzneikunst eingewiesen zu werden. Johann Georg Naumann (1754-1836) war 1787/88 zusammen mit einem Schmied zur Ausbildung in der Tierarzneikunst in Alfort. Er war der Sohn eines sächsischen Postschaffners. Nach einer 6jährige Lehre, während der er in Dresden auch Lehrveranstaltungen über Pferdeheil- und Beschlagskunde besuchte, war er 2 ? Jahre lang Apothekengehilfe. Dann studierte er Medizin, bestand 1783 das Baccalaureatexamen und erhielt nach Vorlesungen über die Notwendigkeit des Studiums der Tierheilkunde die Würde eines Licentiaten (1. akademischer Grad eines Mediziners). In dieser Eigenschaft wurde er dem Grafen von Lindenau von der Leipziger Medizinischen Fakultät für ein Lehramt an der in Berlin zu gründenden "École vétérinaire" empfohlen. Während des Aufenthaltes an der Alforter Veterinärschule stand Naumann mit dem Grafen in brieflichem Kontakt und berichtete über die Ausstattung dieser Einrichtung: Außer einem Wohngebäude und der Kaserne für die Eleven ein sehr schönes zootomisches Kabinett, ein großer Präpariersaal, eine Apotheke und Laboratorium, ein botanischer Garten, eine Schmiede und weitläufige Krankenställe, 
in denen aber nur wenige kranke Pferde standen (Albers 1841, Schrader und Hering 1863, Rieck 1943, Niepage 1988).

Graf von Lindenau hatte 1788 mit Mitteln aus der königlichen Schatulle einen der schönsten Gärten Berlins als Grundstück für die Berliner "École vétérinaire" erworben. Auf diesem nördlich der Weidendammer Brücke zu beiden Ufern des kleinen Flüsschens Panke gelegenen weitläufigen Gelände ließ er alle die von Naumann genannten Gebäude von dem 1788 nach Berlin berufenen schlesischen Baumeister Carl Gotthard Langhans (1732-1808) errichten (Wernicke 2004). Aus dem Situationsplan der Tierarzneischule von 1790 (Abb 2) ist das ersichtlich. Von Zeitgenossen wurde vor allem die von Langhans erbaute (heute unter Denkmalschutz

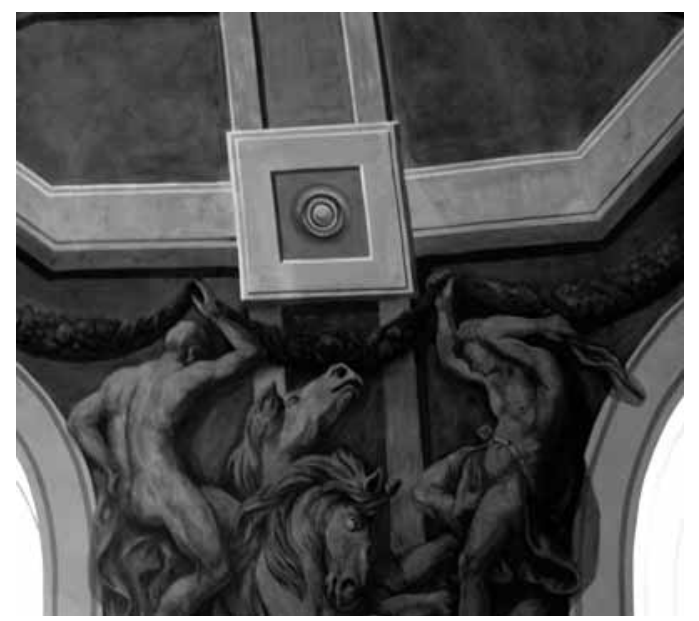

Abb 3 Detail der Malereien in der Kuppelbasis

stehende) "Alte Zootomie" (und ihre malerische Ausgestaltung (Abb 2 und 3) bewundert und in zahlreichen Lithographien und Gemälden dargestellt, darunter auch die in Darstellung des Kunstmalers Ruff (Abb. 4).

So ausgestattet wurde die Berliner Tierarzneischule, nachdem Naumann Anfang 1790 von seiner Ausbildung in Alfort zurückgekehrt war, am 1. Juni 1790 eröffnet. Naumann lehrte jetz† als 1. "Professor der Thierarzneikunde ... den Beschlag, das Exterieur und die innerlichen Krankheiten" der Pferde (Stang und Wirth 1930). Naumann wurde zugleich als "Obergutachter für Pferdekrankheiten in Preussen" bestellt (Rieck 1943 u. 1943 a). Er legte eine Sammlung von Hufeisen sowie Instrumenten und Geräten zur Behandlung normaler und kranker Hufe an, die in der "Alten Zootomie" neben dem "Anatomischen Theater" in besonderen Räumen untergebracht waren (Froehner 1929, Wernicke 2004). Naumanns Veröffentlichungen, Gutachten und Berichte bezogen sich fast ausschließlich auf die "Pferdekenntnis" und "Pferdearzneikunst". Von seinen Lehr- und Handbüchern seien hier das zweiteilige Handbuch "Ueber die vorzüglichen Theile der Pferdewissenschaft" (1. Teil 1800, 2. Teil 1801, 2. Aufl. 1815,3 . Aufl. 1828) und sein "Lehrbuch der Pferdekenntnis" (1822) genannt. Er veröffentliche 1798 die "Beschreibung des Condé, eines alten Leibreitpferdes Seiner Majestät Friedrich des Einzigen ...", unterbreitete u. a. auch Vorschläge zur Bekämpfung der Pferderäude (1799) und der Druse (1820) und unternahm Versuche zur Heilung des Rotzes (Schrader und Hering 1863, Stang und Wirth 1930). Neben Vorlesungen und Übungen für die "Scholaren" der Tierarzneischule hielt er 1799 auch ein erstes Veterinärkolleg vor Kavallerieoffizieren, die aus preussischen Regimentern dazu nach Berlin kommandiert waren. 1810 wurde er Direktor der Tierarzneischule. 1817 wurde er zum Ober-Stabs-Rossarzt des GardeCorps ernannt. Naumann lehrte danach noch bis 1836 und ist am 5. Juni 1836 verstorben (Hertwig 1836 a, Niepage 1988, Wernicke 2004).

Als 2. Professor lehrte Georg Friedrich Sick (1760-1829) ab 1790 Anatomie, Chirurgie, Diätetik (Hygiene) und Seuchenlehre. Sick, der als Candidat der Chirurgie offenbar am Berliner Collegium medico-chirurgicum studiert hatte, wurde 1887 zusammen mit einem Schmied von Graf von Lindenau zur Ausbildung an die 2. Wiener Veterinärschule, das k. k. Tierspital gesandt. In Wien wurde neben der Lehre über Exte-

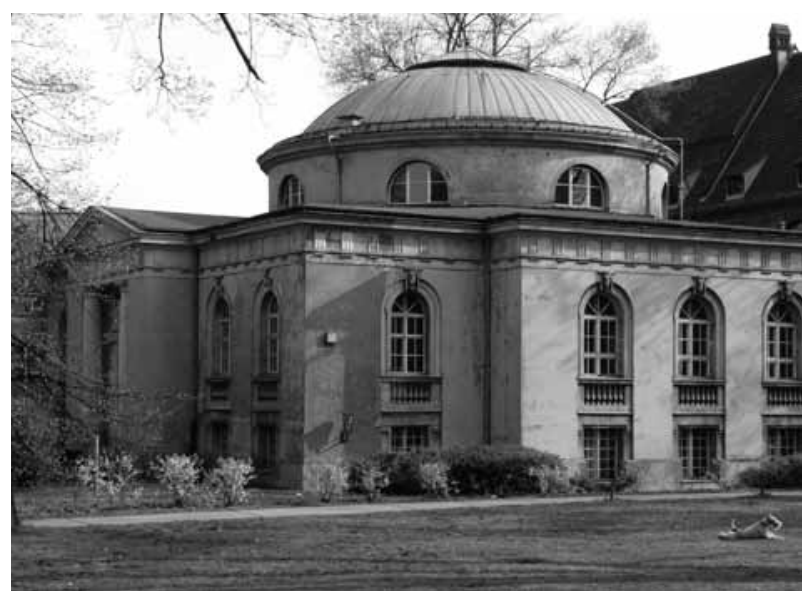

Abb 1 Historische Zootomie im Tierarzneischulgarten der Humboldt-Universität im heutigen Zustand.

rieur, Pferdezucht und Pferdeheilkunde auch die Lehre von den Seuchen und Krankheiten des Hornviehs, der Schafe und der Schweine vorgetragen. Als Sick dann nach 2 ? Jahren Aufenthalt In Wien mit diesem Wissen nach Berlin zurückgekehrt war, entwickelte er sich mehr und mehr zum Spezialisten für die Seuchenbekämpfung. Neben seinem Lehramt wurde er später auf Betreiben landwirtschaftlicher Kreise u. a. zur Bekämpfung der Rinderpest eingesetz† (Schrader und Hering 1863, Stang und Wirth 1931, Rieck 1934). Sein anatomischer Unterricht und die Operationsübungen, die in bzw. vor der "Alten Zootomie" stattfanden, erstreckte sich allerdings hauptsächlich auf Pferde. An den dafür angekauften Pferden wurden zunächst Operationsübungen durchgeführt. Anschließend wurden sie auf einem Platz neben der "Alten Zootomie" getötet, dann in der im Souterrain der "Alten Zootomie" gelegenen "praktischen Anatomie" für Demonstrationen aufbereitet bzw. für Präparierübungen zerlegt (Koch 1965, Wernicke 2004). Zu anatomischen Demonstrationen wurden die Tierkörper oder Tierkörperteile auf einem runden Tisch mit Hilfe einer "Windemaschine" in den Hörsaal, das anatomische Theater befördert. Für die Präparierübungen standen im Souterrain vier heizbare Präpariersäle zur Verfügung, in denen bis zu 60 Eleven gleichzeitig arbeiten konnten. Sick legte auch eine erste anatomische Sammlung an, darunter auch das Skelett des 1804 verstorbenen Leibreitpferdes Friedrich II. Condé (Koch 1965 a, Schröder 1990, Kühn 1994).

Außer seinen Vorlesungen und Übungen versah Sick auch den Außendienst, d. h. er besuchte mit einem Teil der Eleven 
die Marställe in der Breiten Straße, Unter den Linden, in der Dorotheenstraße, wo auch das Garde du Corps stationiert war, und anderen Ortes, um dort kranke Pferde zu behandeln. Nicht zuletzt aus diesem Grunde hatte Graf von Lindenau für die Tierarzneischule einen Standort in der Stadt ausgewählt, von dem aus die Marställe auf dem Fußweg in etwa 15 Minuten zu erreichen waren, und nicht vor den Stadttoren (Wernicke 2003 und 2004).

Große Schwierigkeiten gab es anfangs die Ausbildungskapazitäten der Berliner Tierarzneischule überhaupt auszulasten. Es fand sich nur ein einziger Zivileleve, der bereit war, die Tierheilkunde als zukünftigen Beruf zu erwählen. Daher wurden die Kavallerieregimenter verpflichtet, von jeder Schwadron einen Fahnenschmied zu kommandieren, um ihn als Kurschmied ausbilden zu lassen. Graf von Lindenau bestand ausserdem darauf, dass sogenannte königliche Scholaren für künftige Aufgaben in der Gestütsverwaltung und in den königlichen Marställen als Rossärzte ausgebildet werden.

Wie aus der Tabelle 2 zu ersehen ist, waren bis auf wenige Ausnahmen die Militäreleven in den ersten drei Jahrzehnten in der Überzahl (Schütz 1890, Rieck 1928, Dahmen und Wage-

Tab 2 Die Zahl der Eleven bzw. Scholaren der Berliner Tierarzneischule in den ersten 3 Jahrzehnten ihres Bestehens (zusammengestellt nach Rieck 1928, Wernicke 2003 sowie Materna und Ribbe 1997)

\begin{tabular}{|c|c|c|c|}
\hline Jahr & Militär-Eleven & $\begin{array}{l}\text { Königliche Scholaren bzw. } \\
\text { Obermarstall-Eleven }\end{array}$ & $\begin{array}{l}\text { Freischüler bzw. } \\
\text { Cicil-Eleven }\end{array}$ \\
\hline 1790 & 39 & 6 & 1 \\
\hline 1791 & 39 & 6 & $?$ \\
\hline 1792 & 39 & 7 & 1 \\
\hline 1793 & $40 ?$ & 3 & $?$ \\
\hline 1794 & $40 ?$ & 7 & 1 \\
\hline 1795 & $40 ?$ & 9 & 1 \\
\hline 1796 & 44 & 8 & $?$ \\
\hline 1797 & 44 & 6 & 2 \\
\hline 1798 & 44 & 6 & $?$ \\
\hline 1799 & $40 ?$ & 5 & $?$ \\
\hline 1800 & $40 ?$ & 4 & 4 \\
\hline 1801 & $40 ?$ & 5 & 2 \\
\hline 1802 & $40 ?$ & 5 & 2 \\
\hline 1803 & $40 ?$ & 5 & 1 \\
\hline 1804 & 33 & 4 & 47 \\
\hline 1805 & 39 & 3 & $?$ \\
\hline 1806 & 39 & 3 & $?$ \\
\hline 1807 & 45 & 3 & 9 \\
\hline 1807/08 & \multicolumn{3}{|c|}{ Während der französischen Besatzung ruhte der Schulbetrieb } \\
\hline 1809 & 2 & 2 & 10 \\
\hline 1810 & $?$ & 3 & 2 \\
\hline 1811 & 13 & 1 & ? \\
\hline $1812 / 13$ & \multicolumn{3}{|c|}{ Erneute französische Besatzung } \\
\hline $1814 / 15$ & 49 & 8 & 31 \\
\hline $1816 / 17$ & 38 & 2 & 37 \\
\hline
\end{tabular}

ner 1930, Wernicke 2003). Für die Aufnahme in die Tierarzneischule wurde von ihnen noch 1810 vor allem verlangt, "dass sie das Schmiedehandwerk erlernt haben". "Außer einigen Schulkenntnissen (sollten) sie einigermaßen im Schreiben und Rechnen geübt sein" (Schütz 1890). Das letztere konnten einige der Militäreleven oft nicht nachweisen und so musste extra ein "Kopist" eingestellt werden, der die Militäreleven im
Lesen, Schreiben und Rechnen unterrichtete (Schütz 1890). Dieser geringe Bildungsstand bereitete den noch jungen Professoren große Schwierigkeiten bei der Vermittlung von Kenntnissen (Erdt 1862). So blieb denn die Berliner Tierarzneischule bis 1816 eine reine "Handwerkerschule" (Rieck 1928).

\section{Die erste Schritte auf dem Wege zur wissenschaftlichen Veterinärmedizin (1817 - 1870)}

Als am Beginn des 19. Jahrhunderts das Bürgertum erstarkte, Albrecht Daniel Thaehr (1752-1828) mit seinem Landwirtschaftlichen Lehrinstitut in Möglin aus dem engen Kreis der Empirie herausgetreten war und 1803 neue Bestimmungen für die Seuchenbekämpfung in Kraft getreten waren, geriet die Berliner Tierarzneischule ins Fever der Kritik. Die Tierarzneischule könne und solle mehr leisten, als nur alle drei Jahre für die Armee 40 Fahnen- bzw. Kurschmiede auszubilden und einige Rossärzte für die königlichen Marställe. Bemängelt wurde auch, dass bei der Ausbildung der Hufbeschlag im Vordergrund stand und nur in geringem Maße praktische tierheilkundliche Fertigkeiten vermittelt wurden. Ein Vorschlag, die "Alte Zootomie" auch für die Ausbildung von "Physikern" in der Tierseuchenbekämpfung zu nutzen kam nicht zustande. 1807 wurde die Einbeziehung der "école vétérinaire" mit Professor Naumann in die vom Preussenkönig Friedrich Wilhelm III. (*1770, seit 1797 König, +1840) genehmigte höhere Lehranstalt in Berlin gefordert . 1809 stellte dann Wilhelm von Humboldt (1767 - 1865) den offiziellen "Antrag auf Errichtung der Universität Berlin". Auch er forderte die Einbeziehung des "zootomischen Theaters" als „integranten Teil der Gesamtanstalt" (Müller 1990). Dies stieß allerdings auf den erbitterten Widerstand des Oberstallmeisters von Jagow (Lebensdaten nicht bekannt), der 1808 die Nachfolge von Graf von Lindenau angetreten hatte. Die anhaltende Kritik an den unbefriedigenden Leistungen der Tierarzneischule veranlasste jedoch die Medizinalsektion des preussischen Innenministeriums zu einer Überprüfung. Dazu wurden der Staatsrat Dr. med. Johann Gottfried Langermann (1768 - 1832) und der Professor für Anatomie und Physiologie an der 1810 gegründeten Berliner Universität Dr. phil. et Dr. med. Karl Asmund Rudolphi (1771-1832) eingesetzt. Letzterer war mit den Zuständen an der Tierarzneischule näher vertraut, da er sich bereits 1794, 1796 und $1801 \mathrm{zu}$ Studienzwecken dort aufgehalten hatte, um anschließend an der Universität in Greifswald ein kleines Veterinärinstitut zu leiten. Nach einer eingehenden Überprüfung legten Langermann und Rudolphi 1815 einen Plan zur Reorganisation des Lehrbetriebes vor. Nach Zustimmung des Oberstallmeisters von Jagow, der aber gleichzeitig auf einem Mitspracherecht in den Tierarzneischulangelegenheiten bestand, wurde der Reorganisationsplan verwirklicht. Er trat in Kraft nachdem 1817 die Tierarzneischule aus dem verhängnisvollem Unterstellungsverhältnis unter das Obermarstallamt herausgelöst worden war und Ende des Jahres dem neu gebildeten "Ministerium für geistliche, Unterrichts- und Medizinalangelegenheiten" unterstellt wurde.

Die Tierarzneischule erhielt jetzt für das Sommer- und für das Winterhalbjahr einen festen Studienplan, unterteilt nach Vorlesungen, praktischen Übungen und Repetitionen. Das Lehrpersonal wurde aufgestockt und bestand jetzt aus zwei Professoren, dem Prosektor und dem Apotheker, zwei Lehr- 
schmieden und acht Repetitoren. Die Repetitoren wurden nach dem Vorbild der am k. u. k. Tierarznei-Institut in Wien tätigen Pensions - Tierärzte als neve Lehrkräfte eingeführt. Die Eleven konnten jetzt nicht mehr wie bisher an jedem Tag des Jahres in die Tierarzneischule eintreten, sondern wurden nur noch zur Ostern und zu Michaelis (29. September), d. h. zu Semesterbeginn aufgenommen und mussten nach jedem Semester eine Prüfung ablegen. Nach dreijähriger Ausbildung erfolgte eine Abschlussprüfung und für diejenigen, die sich als Tierärzte für staatliche Aufgaben (Kreistierarzł und Departementstierarzt, Obermarstalltierarzł und Gestütsbeamter) ausbilden lassen wollten, ein Staatsexamen. Mit diesen Maßnahmen begann eine neve Ära an der Tierarzneischule, an der jetzt neben reinen Lehraufgaben auch zunehmend Forschungsaufgaben in Angriff genommen wurden.

1819 wurde der Staatsrat Dr. Langermann als Direktor der Tierarzneischule eingesetzt, behielt aber seine Funktion als vortragender Rat im Ministerium für geistl. usw. Angelegenheiten bei und übernahm so die Verantwortung für die wissenschaftliche Entwicklung der Schule.

1818 erfolgte ein Einteilung der auszubildenden Tierärzte in vier Klassen: 1. Klasse = zukünftige Departementstierärtze; 2 . Klasse $=$ approbierte Tierärzte, die als Kreistierärzte eingesetzt werden konnten; 3. Klasse = Tierärzte mit Schulexamen, die nur eine bedingte Erlaubnis zur Ausübung der Praxis den, galten die Bedingungen von 1790 weiter, d. h. sie wurden von den Regimentern kommandiert und nach der Prüfung zu ihren Regimentern zurückgeschickt. Auch bei den Marstalleleven blieben die alten Bestimmungen in Kraft. Diese Klasseneinteilung hatte bis 1836 Gültigkeit (Erdt 1862).

Jetz† erhielt die Tierarzneischule den Status eines "Wissenschaftlichen Instituts" und die auszubildenden Tierärzte wurden nur noch in zwei Klassen unterteilt. Erst jetzt wurden auch die Anforderungen an die Vorbildung der Eleven näher präzisiert. Eleven, die sich als Tierärzte 1. Klasse ausbilden lassen wollten (approbierte Tierärzte, denen auch amtliche Aufgaben übertragen werden konnten), mussten durch ein Zeugnis nachweisen, dass sie "die zur Versetzung aus der Tertia in die Sekunda eines Gymnasii erforderliche Vorbildung besitzen" (Schütz 1890). Von den Eleven, die sich zu Tierärzten 2. Klasse ausbilden wollen (Tierärzte mit Erlaubnis zur Ausübung der tierärztlichen Praxis), wurde der Nachweis des Besuches niederer Klassen eines Gymnasiums, einer Bürger- oder Mittelschule verlangt. Auch das Zeugnis eines Predigers über gleichwertige Kenntnisse war ausreichend (Schütz 1890). Für die aufzunehmenden Militäreleven, deren Zahl von bisher 40 mit Beginn des Sommersemesters 1832 auf 80 erhöht worden war, sollte die gleiche Vorbildung wie für die Tierärzte 2. Klasse verlangt werden. Sie mussten aber nach wie vor das Schmiede - Handwerk erlernt und den Gesellenstand erlangt haben. So blieb es denn bei ihnen auch jetzt noch beim Stand

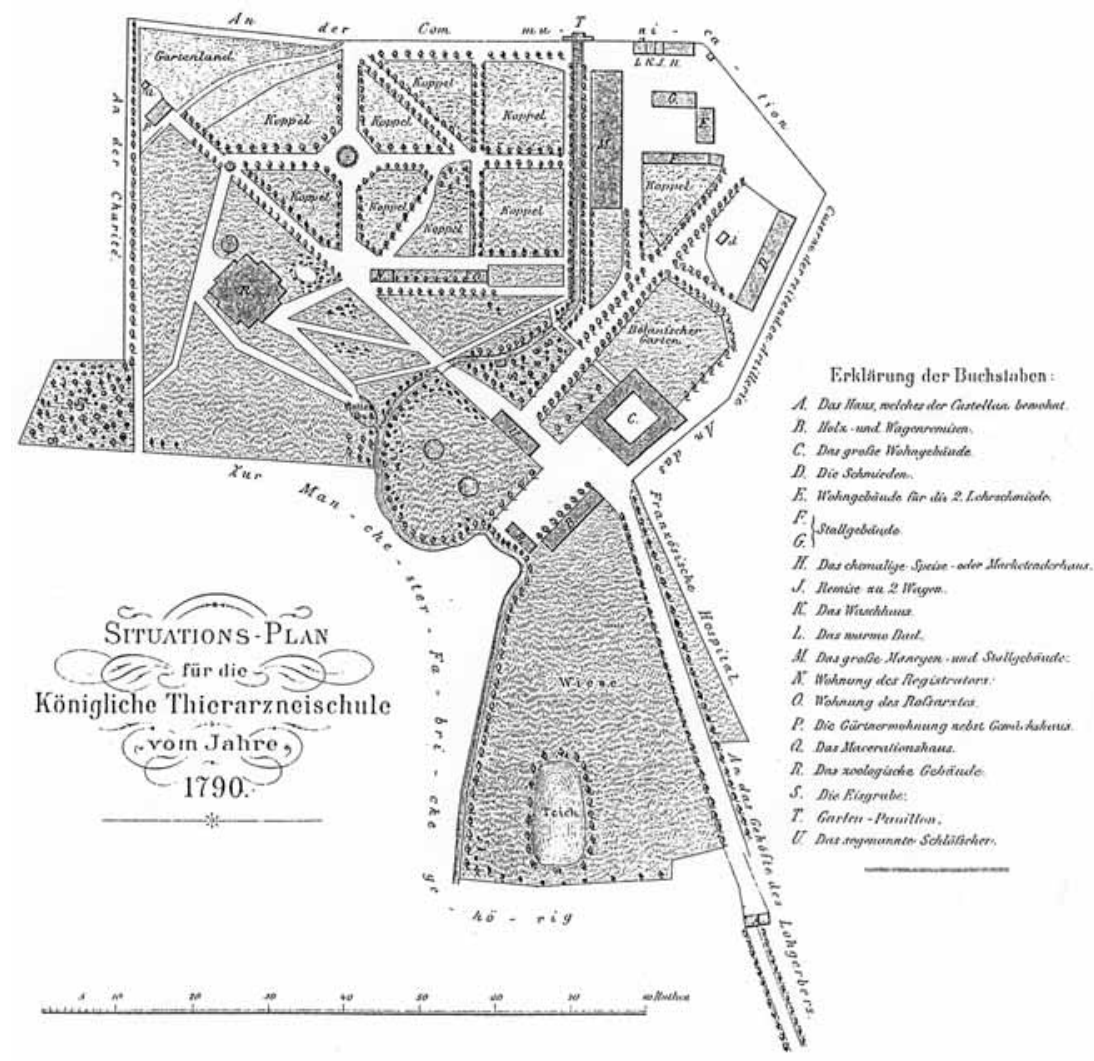

Gebäudeanordnung auf dem Situationsplan der Königlichen Tierarzneischule von 1790 erhielten; 4 . Klasse = geprüfte Schmiede, die als tierärztliche Gehilfen Verwendung finden sollten. Für die Militäreleven, die auch weiterhin an der Tierarzneischule ausgebildet wur- von 1790 (Erdt 1862, Schütz 1890, Schmaltz 1936). 1819 wurde des Lehrpersonal um zwei Obertieraztstellen erweitert und im gleichen Jahr wurde Ernst Friedrich Gurlt 
(1794-1882), der in der Folgezeit als Veterinäranatom das wissenschaftliche Profil der Tierarzneischule mitbestimmte, als Repetitor angestellt. Ihm folgte 1823 Carl Heinrich Hertwig (1798-1881), der sich später als Veterinärchirurg und Kliniker um die wissenschaftliche Entwicklung der Berliner Tierarzneischule bleibende Verdienste erwarb.

Gurlt hatte wie Naumann zunächst den Apothekerberuf erlernt, dann in Breslau Medizin studiert, war dann Prosektor an der Medizinischen Fakultät und wurde dort zum Dr. med. promoviert. Als er 1819 nach Berlin kam, wurden ihm zunächst die Vorlesungen über Botanik und die botanischen Exkursion als Lehrfach zugewiesen. Den Anatomen Johann Dietrich Reckleben (1785-1851) hatte er bei den anatomischen Übungen in der "Alten Zootomie" zu unterstützen und musste Repetitionen in der Osteologie, Anatomie und Physiologie durchführen. 1821 unternahm er eine Studienreise zur Ausbildung in der Tierheilkunde, die ihn nach Wien und nach Süddeutschland führte. 1824 übernahm Gurlt von Reckleben die Vorlesungen über normale und pathologische Anatomie sowie über Physiologie. 1825 wurde er als Oberlehrer angestellt. Er las jetzt auch über Allgemeine Naturgeschichte, leitete in der "Alten Zootomie" die zootomischen Übungen und überwachte die Sektionsübungen, nahm aber selbst keine Sektionen vor, sondern liess diese von den Studierenden ausführen. Zeitweilig las er auch Allgemeine Pathologie und Therapie. 1826 wurde er zum Professor ernannt und von 1849 bis 1870 war in Gemeinschaft mit dem Ober-Inspektor der Charité Rechnungsrat Dr. jur. Karl Heinrich Julius Esse (1808 -1874) Direktor der Tierarzneischule. "Gurlt war ein Meister in der praktischen Anatomie, er präparierte schnell und ungemein sauber, er liess sich bis zum Ende seiner Lehrtätigkeit ... nicht nehmen, die Präparate für die Vorlesungen eigenhändig anzufertigen" (Müller 1890). Unter seinen Händen ist die Sammlung der anatomischen und pathologisch-anatomischen Präparate, einschließlich zahlreicher Missbildungen auf über 6.500 angewachsen. Dafür wurde, nachdem 1840 das neve Hauptgebäude der Tierarzneischule an der Luisenstraße entstanden war (Abb 6), in der "Alten Zootomie" zusätzlich Platz geschaffen, indem man die Hufeisen- und Instrumentensammlungen in den Neubau verlagerte.

Seine Präparate bildeten auch die Grundlage für vergleichende Untersuchungen. So wurde Gurlt zum Schöpfer der vergleichenden Anatomie der Haustiere, für die er bereits 1822 ein international beachtetes Handbuch herausgab, dem 4 Auflagen folgten. 1860 ergänzte er sein zweibändiges Werk durch einen Hand-Atlas. Ausgangspunkt seiner vergleichenden Untersuchungen und Darstellungen war und blieb aber das Pferd. So enthalten z. B. die 22 Figuren seines Handatlanten ausschließlich Darstellungen der anatomischen Verhältnisse beim Pferd (Gurlt 1860). Auch in der fünften von seinen Schülern August Gottlob Theodor Leisering (1820 1892) und Carl Friedrich Müller (1825 - 1901) bearbeiteten Auflage des Handbuches stellen von den 171 Abbildungen 127 die Verhältnisse beim Pferd dar (Leisering und Müller 1873).

Zusätzlich zu seinem Handbuch schrieb Gurlt 1832 auch eine Anatomie des Pferdes. Weitere Werke folgten, darunter die "Chirurgische Anatomie und Operationslehre für Tierärzte", die er 1847 zusammen mit Hertwig herausgab. Zusammen mit Hertwig gab er auch von 1834 - 1874 die erste wis- senschaftliche Zeitschrift der Veterinärmedizin, das "Magazin für die gesammte Thierheilkunde" heraus. Hier veröffentlichte er auch die meisten seiner über 130 Publikationen (Schrader und Hering 1863, Stang und Wirth 1928, Gerber 2004). Hertwig, der Mitbegründer der ersten wissenschaftliche Periode der Berliner Tierarzneischule, hatte seit 1817 in Breslau am Königlichen Chirurgischen Institut Arzneikunde studiert. Ab 1820 hat er dann mit Unterstützung der schlesischen Regierung 3 Semester lang in Wien und dann auch in München Tierheilkunde studiert. Anschließend unternahm er eine Studienreise durch Deutschland, die Schweiz, nach Frankreich, England und Holland. Nach seiner Berufung als Repetitor an die Berliner Tierarzneischule wurden ihm ab Sommersemester 1824 die Vorlesungen über Allgemeine Chirurgie, die Leitung des Spitals für kleine Haustiere und die Repetitionen in der Arzneimittellehre übertragen. Ab Wintersemester 1824/25 übernahm er auch die Vorlesungen über Spezielle Chirurgie und Operationslehre sowie die Repetitionen über die gesamte Therapie und über spezielle Chirurgie und Operationslehre. 1826 übernahm er auch die Leitung und Überwachung der Operationsübungen. Mit dem Eintritt in das Spital für große Hautiere, dessen alleinige Leitung er ab 1832 von Naumann zusammen mit dem gesamten klinischen Unterricht übernahm, profilierte sich Hertwig als der bedeutendste Kliniker seiner Zeit.

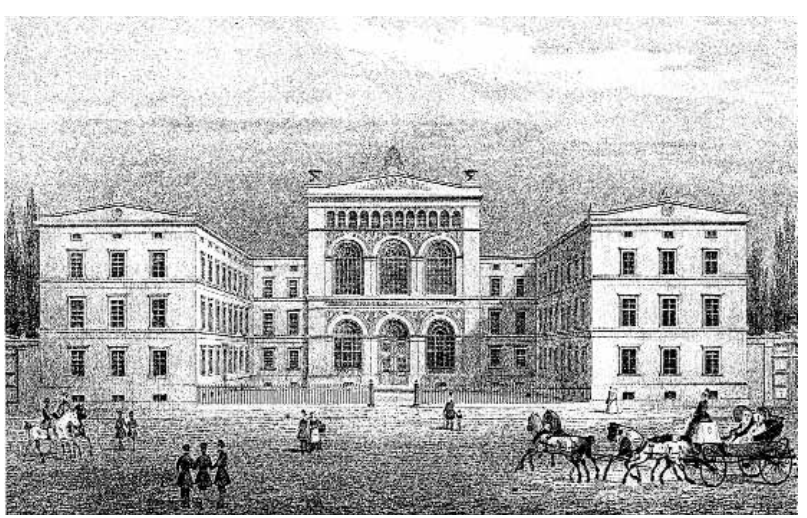

Abb 6 Hauptgebäude der Tierarzneischule von 1840

Hertwigs wissenschaftlicher Ruhm begann nach seiner 1826 erfolgten Promotion zum Dr. med. an der Berliner Universität, nach der Erlangung der Approbation als Arzt (1827), nach seiner Ernennung zum Obertierarzł (1828) und zum Oberlehrer (1829) mit der Veröffentlichung einer Schrift über die Hundetollwut, die 1829 mit einem Vorwort von Christoph Wilhelm Hufeland (1762-1836) erschien. 1833 wurde er dann zum Professor ernannt.

Hertwigs wissenschaftliches Interesse galt vor allem der Veterinärchirurgie. Er schrieb 1847 zusammen mit Gurlt die Chirurgische Anatomie und Operationslehre für Tierärzte und verfasste ein Praktisches Handbuch der Chirurgie für Tierärzte (1850, 2. Aufl. 1874). Außerdem erschien von ihm ein Taschenbuch der gesamten Pferdekunde (1851, 2. Aufl. 1878) und ein Buch über "Die Krankheiten der Hunde und deren Heilung" (1853, 2. Aufl. 1880) (Schrader und Hering 1863, Stang und Wirth 1928, Gerber 2004). Neben weiteren Publikationen veröffentlichte Hertwig im "Magazin für die gesammte Thierheilkunde" "Berichte über die Clinik in der Königlichen Thierarzneischule zu Berlin", aus denen zu entnehmen ist, dass die überwiegende Mehrzahl der zur Behand- 
lung und Beurteilung oder zu anderen Zwecken aufgenommenen Patienten Pferde waren: Während es vor 1819 jährlich nur 300 bis 450 Tiere waren, stieg ihre Zahl 1820 auf 678, 1825 auf 1225,1830 auf 1.495 und 1835 auf 1.972 an. 1839 waren es dann sogar 2.424 Pferde, die stationär aufgenommen wurden. Außerdem wurden an der Tierarzneischule auch noch zwischen 3.000 und 4.000 Pferde ambulant behandelt oder begutachtet. Rinder, Schafe und Schweine kamen kaum zur Behandlung. 1839 waren es lediglich 6 Rinder, 3 Schafe und 2 Schweine. Aus diesen Zahlen lässt sich wohl ableiten, dass unter dem Kliniker Hertwig fast ausschließlich Pferdeheilkunde gelehrt wurde (Hertwig 1836, 1837 u. 1840).

Betrachtet man nun auch die Entwicklung der Zahl der Eleven, die an der Tierarzneischule ausgebildet wurden, so entsprach dies vor allem den Erfordernissen ihrer zukünftigen Einsatzgebiete. So ergaben sich für die Periode von 1817 bis 1870 folgende Zahlen: In 53 Semestern lag die Anzahl der Zivileleven bei durchschnittlich 73, diejenige der Militäreleven lag bei durchschnittlich 58. Zu Beginn der 30er Jahre kehrte sich dann das Verhältnis um und nun übertraf in 54 Semestern die Zahl der Militäreleven diejenige der Zivileleven z. T. um mehr als das Doppelte, im Durchschnitt 94 Militäreleven und 52 Zivileleven. Neben diesen beiden Gruppen von Studierenden gab es auch die sog. Hospitanten. Ihre Zahl belief sich durchschnittlich auf jährlich etwa 14 Personen (Offiziere, Landwirte, Ausländer etc.) (Schütz 1890).

Die verstärkt einsetzende Ausbildung von zukünftigen Militärtierärzten erforderte auch strukturelle Veränderungen an der Tierarzneischule. So erhielt 1849 die Unterkunft der Militäreleven im Südflügel des Hauptgebäude in der Luisenstraße die Bezeichnung "Militär-Kurschmiede-Eleven-Institut". Die Angehörigen dieses von der Tierarzneischule mehr oder weniger unabhängigen Instituts waren Armeeangehörige und unterstanden jetzt der Aufsicht eine Offiziers, während sie 1790 zunächst nur von zwei Wachmeistern überwacht wurden. Sie studierten aber als Militäreleven weiter an der Tierarzneischule. 1862 wurde das "Militär-Kurschmiede-Eleven-Institut" durch Kabinetsordre in eine "Militärrossarztschule" umgewandelt und nun von einem militärischen Direktor geleitet, der für die militärische Ausbildung der Eleven verantwortlich war. Erster Direktor dieser Militärrossarztschule war der Rittmeister Oscar von Diebitsch (1824 - 1906) (Schmaltz 1936).

1868 wurde auf einem vom Tierarzneischulgarten abgezweigten Grundstück an der Karlstrasse (jetzt Reinhardtstrasse) für eine Militär-Lehrschmiede unter der Leitung des früheren Marstalltierarztes Friedrich Dominik (1829 1891) ein Gebäudekomplex in Betrieb genommen, in dem nun auch die Eleven der Militärrossarztschule untergebracht wurden. In dieser neuen Einrichtung wurden jetzt die Beschlagschmiede für die Armee ausgebildet und die zukünftigen Militäreleven mussten hier vor der Aufnahme ihres Studiums an der Tierazneischule ein halbes Jahr lang Dienst tun (Schütz 1890, Ohmke 1940, Krokotsch 1991).

Die ständig steigenden Patientenzahlen erforderten auch bauliche Veränderungen an der Tierarzneischule. Bereits 1837/38 wurden die alten inzwischen baufällig gewordenen Pferdeställe abgerissen und an ihrer Stelle der größte Teil der Pferdeställe, in denen der klinische Unterricht stattfand, neu erbaut.
Zusammenfassend lässt sich demnach für die erste wissenschaftliche Periode der Berliner Tierarzneischule feststellen: Das Lehrpersonal wurde vermehrt (1869/70 $=4$ Titularprofessoren und 3 etatmäßige Lehrer), begann sich wissenschaftlich zu betätigen und begründete mit zahlreichen Publikationen den Ruf der Berliner Tierarzneischule als "Wissenschaftliches Institut"; die Patientenzahlen, vorwiegend Pferde, stiegen um mehr als das Zehnfache an, woraus sich ergab, dass vorwiegend Pferdeheilkunde gelehrt wurde; die Anzahl der Studierenden nahm ständig zu, wobei die Zahl der Militäreleven mehr als verdoppelt wurde.

Veterinärmedizinische Lehre und Forschung im Deutschen Kaiserreich bis zum Ende des I. Weltkrieges (1871 -1918)

Die eigentlich naturwissenschaftliche Periode der Berliner Tierarzneischule begann mit der Berufung von Andreas Christian Gerlach (1811-1877) am 1. April 1870 als alleiniger wissenschaftlicher und administrativer Direktor. Seine ersten Schritte waren die Neuaufteilung der Lehrfächer, die Neuordnung des Lehrplanes und sein energischer Kampf für die Erhöhung der Vorbildung der Eleven, die dann ab 1882 als Studierende bezeichnet wurden. Das bisherige Lehramt von Gurlt, der sowohl die normale als auch die pathologische Anatomie sowie die Physiologe gelehrt hatte, wurde zunächst zweigeteilt. Carl Friedrich Müller (1825-1901), der bereits seit 1862 etatmäßiger Lehrer war und neben seiner Tätigkeit in der Anatomie bei Gurlt Vorlesungen über Exterieur, Gestütskunde und Diätetik (Hygiene) sowie klinische Demonstrationen übernommen hatte, erhielt die normale Anatomie und hatte zunächst auch noch die Physiologie zu lehren. Ausserdem lehrte er noch Zoologie und Botanik. Der Virchowschüler Johann Wilhelm Schütz (1839-1920) hatte die Gewebelehre/Histologie vorzutragen, leitete eine Zeit lang die Kleintierklinik und erhielt ab Wintersemester 1870/71 die volle Verantwortung für die pathologische Anatomie und die Allgemeine Pathologie. 1876 wurde dann für die Physiologie auch ein eigener Lehrstuhl geschaffen, der mit dem Schüler von Emil Heinrich Du Bois-Reymond (1818-1896) Hermann Munk (1839-1912) besetzł wurde. Munk übernahm zunächst auch die Histologie. Schließlich wurde 1876 noch ein eigenständiger Lehrstuhl für Chemie errichtet, der mit dem Chemiker Adolf Pinner (1842-1909) besetzt wurde. Auf Antrag von Gerlach wurden zu klinischen Lehrern auch noch 1870/71 Wilhelm Dieckehoff (1835-1903), 1874 Heinrich Möller (1841-1932) und 1876 Albert Friedrich Christian Eggeling (1848-1920) berufen bzw. angestellt.

Zunächsł mussten sich Müller und Schütz mit den räumlich beschränkten Verhältnissen in der "Alten Zootomie" abfinden. Gerlach ließ dann für die Pathologie an die "Alte Zootomie" einen Anbau anfügen, der auf der Hörsaalebene einen Übergang zu den neu eingerichteten Räumen mit den pathologisch - anatomischen Präparaten erhielt, die jetzt hier zu Lehrzwecken untergebracht wurden. Im Souterrain dieses "Gerlachanbaus" befanden sich ein Sektionsraum mit 4 Sektionstischen. Als dann Munk berufen wurde, erhielt er zunächst zwei Räume in der "Alten Zootomie", in denen er ein Laboratorium einrichtete. Müller und Munk konnten ab 1883 den "Gerlachanbau" mitbenutzen, da Schütz einen repräsentativen Neubau für das Pathologische Institut der Tierarzneischu- 
le erhalten hatte. Zu ihrer Unterstützung wurde 1886 Reinhold Schmaltz (1860-1945) als Prosektor in der Anatomie und ab 1887/88 als Lehrer für Gewebelehre/Histologie, die zwischenzeitlich von Munk gelehrt wurde, angestellt. 1890 wurden dann die Sammlungsräume im "Gerlachanbau" zum Experimentierhörsaal für den Chemiker Pinner hergerichtet, so dass jetzt in der "Alten Zootomie" zusammen mit dem "Gerlachanbau" Lehrveranstaltungen auf den Gebieten Anatomie, Histologie, Physiologie und Chemie stattfanden. Erst nachdem Schmalz 1891 Titularprofessor geworden war und er 1896 Nachfolger von Müller wurde, begannen die Vorbereitungen für den Neubau eines großzügig eingerichteten Instituts der Anatomie, das aber erst 1902 fertiggestellt war. Hier vertrat Schmaltz dann als Alleinvertreter die Anatomie, Histologie und Embryologie. Die ursprüngliche Funktion der "Alten Zootomie" als Lehr- und Forschungsstätte für die Anatomie der Haustiere wurde hierdurch endgültig aufgehoben. Es folgte ein Zeitabschnitt von 12 Jahren, in dem das Gebäude von den Physiologen und Chemikern genutzt wurde. Nach dem I. Weltkrieg wurde die "Alte Zootomie" mit dem "Gerlachanbau" unter Denkmalsschutz gestellt und für das 1912 neu errichtete Ordinariat für Nahrungsmittelkunde als Lehrund Forschungsstätte hergerichtet (Fröhner 1950, Nusshag 1959/60).

Diese Entwicklung ging konform mit den stetig wachsenden Zahlen der Studierenden. Vom Wintersemester 1870/71 bis zum Wintersemester 1918/19 studierten in 63 Semestern, wo die Zahl der Zivil-Studierenden mehr als doppelt so hoch war wie die der Militär-Studierenden, durchschnittlich 274 Zivilisten pro Semester und 122 Militärangehörige. In 34 Semestern war die Zahl der Militär-Studierenden mit durchschnittlich 109 mehr als doppelt so hoch wie die der Zivil-Studierenden mit durchschnittlich 49. In den Jahren 1896/97 bis 1914 waren teilweise pro Semester mehr als 500 Studierende immatrikuliert. Das Studium der Veterinärmedizin wurde vor allem für junge Leute attraktiv, nachdem die Tierarzneischule 1887 durch den preussischen Minister für Landwirtschaftliche Angelegenheiten ein Statut als Tierärztlichen Hochschule mit neun Lehrerstellen erhalten hatte. Auch die Abschaffung der tierärztlichen Zweiklasseneinteilung verbunden mit der Abschaffung des Nachweises einer Schmiedelehre sowie die Einführung der Hochschulreife (1902/03)als Vorbildung haben dazu beigetragen. Die Tierärztliche Hochschule, der 1903 das Rektoratswahlrecht verliehen wurde und der 1910 auch das Promotionsrecht und 1918/19 das Habiltationsrecht eingeräumt worden war, hatte nun ihre vollen Würden als akademische Lehranstalt erreicht (Schmaltz 1912, 1936).

Auch für die Militär-Studierenden waren bereits 1869 höhere Anforderungen an die Vorbildung gestellt worden. Für sie wurde 1903 die "Militär-Veterinär-Akademie" gegründet, die aus der Militär-Rossarztschule hervorging und in den Gebäuden auf dem Gelände an der Karlstrasse eingerichtet wurde. Für die Aufnahme in diese Veterinärakademie war auch das Abiturzeugnis Voraussetzung. Die "Veterinär-Aspiranten" mussten außerdem mindestens 6 Monate bei der Truppe gedient haben. Sie wurden dann für 6 Monate an die Militär-Lehrschmiede Berlin kommandiert und studierten danach als Militärangehörige im Beurlaubtenstand 7 Semester an der Tierärztlichen Hochschule. Nach Ablegung der tierärztlichen Fachprüfung wurden sie einem Truppenteil überwiesen und als Unterveterinär wieder für 6 Monate an die Lehrschmiede kommandiert, der auch eine Pferdeklinik angegliedert war. Der bisherige vierwöchige Lehrschmiedekurs entfiel jedoch. Danach hatten sie für jedes Semester eine Dienstpflicht von einem Jahr als Militär-Rossarzt bzw. ab 1910 als Veterinäroffizier abzuleisten. 1919 wurde die "Militär-Veterinär-Akademie" nach den Bestimmungen des Vertrages von Versailles aufgehoben (Schmaltz 1936, Ohmke 1940).

Für die Entwicklung der Pferdeheilkunde war Wilhelm Schütz die zweifellos markanteste Persönlichkeit. Er hatte als einer der ersten Zivileleven mit Abitur ein Studium an der Berliner Tierarzneischule absolviert und 1860 mit einem sehr guten Staatsexamen abgeschlossen. Anschließend studierte er von 1860 bis 1864 an der Berliner Universität Medizin. Zwischendurch bestand er 1862 die Prüfung als Kreistierarzt mit dem Prädikat "Sehr gut". Von 1864 bis 1866 war er Kreistierarz† in Ostpreussen. 1866 nahm er als Rossarzt am preussisch österreichischen Krieg teil und war zugleich als Militärarzt in einem Feldlazarett tätig. Nach Rückkehr aus dem Krieg war er Volontär an der Tierarzneischule und bis 1868 Assistent von Rudolf Virchow, bestand 1867 das Examen als Departementstierarzt, erhielt 1867 eine Repetitorenstelle an der Tierarzneischule und wurde 1868 mit einer bei Virchow angefertigten Dissertation zum Dr. med. et. chir. promoviert. Bis 1870 war er Assistent bei Gurlt, Hertwig und Werner Theodor Johann Spinola (1802-1872). 1870 erhielt er die 5. Lehrerstelle an der Tierarzneischule, mit der ihm bis 1876 die Lehrveranstaltungen und Übungen auf dem Gebiet der "Gewebelehre und mikroskopischen Uebungen" übertragen wurden. Am 1. Oktober 1870 übertrug ihm der Tierarzneischuldirektor Gerlach das nunmehr selbständige Lehramt für pathologische Anatomie und Allgemeine Pathologie. Dies war die Geburtsstunde des ersten pathologischen Lehrstuhles an einer Tierarzneischule.

Am 22. Oktober 1870 führte Wilhelm Schütz in aller Vollständigkeit seine erste Sektion bei einem Pferd noch unter freiem Himmel aus und begann damit die von ihm bis an sein Lebensende geleiteten "Sektions-Übungen" für die Studierenden. Er übertrug dabei die bei Virchow erlernte Sektionstechnik auf die anatomischen Verhältnisse beim Tier. 1879 entwarf er die "Anweisung für das Obduktionsverfahren bei ansteckenden Krankheiten der Hausthiere", die den späteren Tierseuchengesetzen als Anlage beigefügt wurde und Prinzip heute nach Anwendung findet. In grenzenloser Verehrung für seinen Lehrer Virchow, mit dem er zusammen 1882 auch Untersuchungen über die pathologische Anatomie des Pferderotzes unternahm, übernahm er dessen Zellularpathologie und veröffentlichte bereits 1872 eine Schrift mit dem Titel "Die Lehre von der Constitution vom cellularpathologischen Standpunkte".

Anders als sein verehrter Lehrer Virchow suchte er auch den Kontakt zu Robert Koch (1843-1910). Er wurde 1880 zur Mitarbeit in der bakteriologischen Abteilung des Kaiserlichen Gesundheitsamtes herangezogen, die sich von 1880 bis 1885 im Nachbargebäude der Tierärztlichen Hochschule in der Luisenstrasse 57 befand. Hier erlernte Schütz die bakteriologischen Techniken und hier begann auch eine intensive Zusammenarbeit mit Kochs ersten Assistenten Friedrich Loeffl er (1852-1915) (Schneck 1997, Toellner 2000). Loeffler und Schütz entdeckten 1882 den Erreger der Rotzkrankheit und 
isolierten auch den Erreger des Schweinerotlaufes. 1882 berichtete Schütz auch über das Vorkommen des Rotlaufs beim Pferd. Nachdem Schütz dann in seinem neven Pathologischen Institut auch ein bakteriologisches Laboratorium eingerichtet hatte, isolierte er 1887 auch den Streptococcus equi als Erreger der Druse. Die Erfolge seiner bakteriologischen Forschungen waren dann Veranlassung, ab Wintersemester 1889/90 "Bakteriologische Übungen" in den Lehrplan der Berliner Tierärztlichen Hochschule aufzunehmen (Schütz 1890).

1891 begann Schütz dann auch zusammen mit Emil Adolf von Behring (1854-1917) und Shibasaburo Kitasato (18521931) Versuche zu Tetanusimmunisierung von Pferden und anderen Tieren. Im Vordergrund seiner Forschungen stand aber bis zu seinem Lebensende der Pferderotz. 1894 unternahm er Versuche mit dem von den russischen Tierärzten Christofor Ivanovic Gel'man (1848-1902) und Otton Ivanovic Kal'ning (1856-1891) 1891 entwickelten Mallein zur Rotzdiagnostik (Skrjabin 1969 u 1972). 1914 entwickelte er die "Komplementablenkungs-Hämagglutinationsprobe" zur Rotzdiagnostik, mit deren Hilfe es gelang, die Pferdebestände der deutschen Armeen während des I. Weltkrieges weitge-

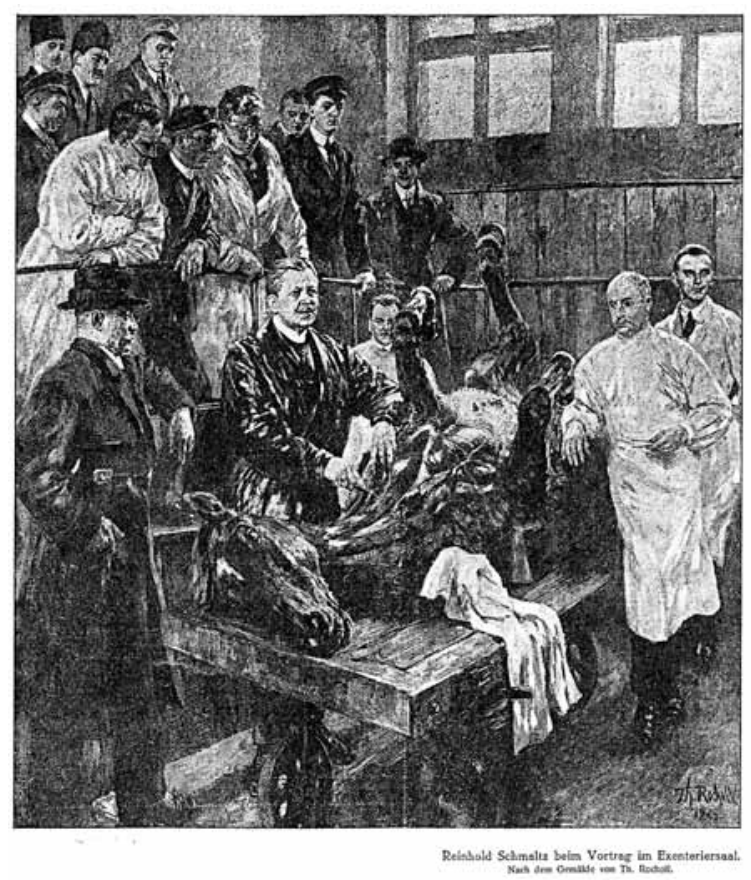

Abb 6 Reinhold Schmaltz (1860-1945) bei einer anatomischen Demonstration an einem Pferdekörper. Nach einem Gemälde von Th. Rocholl 1925

hend von der gefährlichen Rotzerkrankung freizuhalten. In seiner letzten 1920 zusammen mit Otto Waldmann (18951955) verfassten Veröffentlichung berichtete er noch über „Versuche zur aktiven Immunisierung gegen die Rotzkrankheit der Pferde". Für seine bahnbrechenden Arbeiten auf den Gebieten der Veterinärpathologie und -bakteriologie wurde Wilhelm Schütz vielfach geehrt und seine Freunde, Mitarbeiter und Schüler setzten ihm im Hochschulgarten 1922 ein Denkmal (Bergmann und Wenzel 1990, Wernicke 2000, 2000 a, Gerber 2004).

Für die Pferdeheilkunde erlangten auch der Kliniker Dieckerhoff, der Chirurg Möller und der Anatom Schmaltz Bedeu- tung. Alle drei stellten das Pferd in den Mittelpunkt ihrer Lehrveranstaltungen und Forschungsarbeiten.

Wilhelm Dieckerhoff hatte sich nach dem Studium in Berlin und der Ableistung seines Militärdienstes bei einem Garde - Ulanen-Regiment 1858 als praktischer Tierarzt in Bochum niedergelassen, wo er neben seiner tierärztlichen Tätigkeit für die Verbesserung der einheimischen Vieh- und Pferdeschläge wirkte und u. a. mehrfach in Frankreich Hengste einkaufte. Nach seiner Ernennung zum Lehrer an der Berliner Tierarzneischule übernahm er neben seiner Tätigkeit in der Klinik die Vorlesung über Exterieur der Pferde und Gestütskunde sowie in den Wintersemestern 1871/72 und 1873/74 auch die Vorlesung über Hufbeschlag sowie die Leitung der Operationsübungen. Ab 1878 übernahm er die Leitung der Klinik für grosse Haustiere und der Poliklinik für Pferde. In der Klinik von Dieckerhoff wurden nach wie vor hauptsächlich Pferde behandelt. Sie verfügte 1890 für rund 40 Pferde über folgende Räumlichkeiten: 13 Laufställe, 24 Kastenstände, 3 isoliert gelegene Einzelställe, 1 Demonstrationshalle und eine Reitbahn. Unter den von Diekkerhoff veröffentlichten insgesamt 159 Werken, Einzelveröffentlichungen und Gutachten befassten sich allein 91 mit dem Pferd, seinen Krankheiten und Gebrechen. Dieckerhoff kann daher wohl mit Recht als gefragter Pferdekenner und Spezialist der Pferdeheilkunde angesehen werden. Nach seinem Tode wurde ihm 1904 im Hochschulgarten ein Denkmal gesetzt (Wernicke 2000, Gerber 2004).

Der Chirurg Dr. phil. Heinrich Möller wurde vor allem bekannt durch die Einführung der Asepsis und Antisepsis in die Veterinärchirurgie. Besondere Verdienste erwarb er sich, indem er der Diagnose der Lahmheit durch Trennung in Hang- und Stützbeinlahmheit eine sichere Grundlage gab. Aufsehen erregten auch seine Kehlkopfoperationen, mit denen er den Versuch unternahm das Kehlkopfpfeifen der Pferde zu beseitigen. Er führte seine Operationen noch unter freiem Himmel durch, da es zu seiner Zeit an der Tierärztlichen Hochschule noch keine Operationshalle gab. Auch seine Spat-Operation wurde seinerzeit gelobt. Schließlich gab er zusammen mit seinem Schulfreund Born (Lebensdaten nicht bekannt) eine "Pferdekunde" heraus, die in Offizierskreisen weite Verbreitung fand. Er verfasste auch eine erstes auf wissenschaftlicher Grundlage stehendes Lehrbuch über "Hufkrankheiten", das in vielen Auflagen erschien. Möller gab, inzwischen als Veterinärchirurg international bekannt und geehrt, seine Lehrtätigkeit an der Tierärztlichen Hochschule 1895 auf, da er sich bei der Neuvergabe des Rektorat übergangen sah (Bongert 1931).

Reinhold Schmaltz der nach seiner Schulausbildung zunächst ein Offizierslaufbahn einschlug (1878-1882), danach in Hannover Tierheilkunde studierte und nach zweijähriger Praxistätigkeit 1886 als Prosektor seine Karriere als Anatom und Histologe an der Berliner Tierarzneischule begann, hatte neben seinen vielfältigen Aktivitäten als Standespolitiker die Veterinäranatomie durch zahlreiche Werke über die Anatomie des Pferdes bereichert. Er schrieb u. a. eine "Anatomie des Pferdes" (1888, 2. Auflage 1919, 3. Auflage 1928), verfasste ein dreibändiges Werk über "Präparierübungen am Pferd" (1898 bis 1903, 2. Auflage 1910 bis 1913) und einen "Atlas der Anatomie des Pferdes" in 5 Bänden, von denen die einzelnen Bände ab 1901 in bis zu 3 Auflagen erschienen. Seine Anatomie war demnach im wesentlichen eine Pferdeana- 
tomie und auch in der Lehre demonstrierte er die Anatomie vorwiegend am Pferd (Lothes 1925, Gerber 2004).

Für die naturwissenschaftliche Entwicklungsperiode der Berliner Tierarzneischule zur vollakademischen Tierärztlichen Hochschule lässt sich wohl resümierend feststellen: Sie begann mit dem Antritt von Andreas Christian Gerlach als Tierarzneischuldirektor, dessen Denkmal im Tierarzneischulgarten steht, im Jahre 1870 und endete 1919 mit der Verleihung des Habilitationsrechtes für die Tierärztliche Hochschule. Sie begann mit 5 Lehrerstellen und endete mit 11 Ordinariaten. Die von Naturwissenschaftlern und Medizinern im 19. Jahrhundert erworbenen Kenntnisse wurden aufgenommen, in den Lehrplan integriert und für die veterinärwissenschaftliche Forschung nutzbar gemacht, was auch der Pferdeheilkunde zugute kam.

\section{Veterinärmedizinische Lehre und Forschung in der Wei- marer Republik und im "Dritten Reich" (1919 - 1945)}

Nach dem I. Weltkrieg verringerte sich zunächst die Zahl der Studierenden, insbesondere durch den Wegfall der Militärstudierenden. In einem Zwischensemester und im Sommersemester 1919 studierten noch einmal 271 Militärangehörige, dann aber wurden nur noch Zivilisten zum Studium zugelassen. Bis zum Sommersemester 1931 belief sich die Zahl der Studierenden auf durchschnittlich 264 pro Semester (Dahmen und Wagener 1930). Trotz dieses Rückganges der Studentenzahlen wurde die Zahl der Ordinarien während der Weimarer Republik auf 13 erhöht. Gleichzeitig wurde durch Neuund Umbauten das Lehrangebot verbessert und für die Forschung ergaben sich erweiterte Möglichkeiten.

Neben der für das Institut für Nahrungsmittelkunde umgebauten "Alten Zootomie" erhielt die Poliklinik für große Haustiere 1920 einen Neubau, zu dem auch eine Schmiede gehörte (Abb 7). 1926 wurde diese Poliklinik in eine Medizinische und eine Chirurgische Poliklinik mit Schmiede unterteilt und den beiden stationären Kliniken zugeordnet. Die Chirurgische Tierklinik konnte 1926 einen Neubau mit Operationssaal und Röntgenstation beziehen. In dem nun frei gewordenen Gebäude der Poliklinik wurde im gleichen Jahr das erste Parasitologische Institut an einer deutschen veterinärmedizinischen Ausbildungsstätte eingerichtet. In der ersten Etage der Chirurgischen Tierklinik fand auch das bereits 1923 neu eingerichtete 12. Ordinariat für Tierzucht sein Domizil. 1928 wurde als 13. Ordinariat der Lehrstuhl für Tierseuchentherapie gegründet. Das zu diesem Ordinariat gehörende "Institut für Tierseuchentherapie" wurde zunächst im 2. Stock des Hygieneinstituts untergebracht. 1929 wurde für das Institut für Veterinärpathologie eine neverbaute Sektionshalle in Betrieb genommen. Daneben erhielt das "Institut für Parasitenkunde und veterinärmedizinische Zoologie" ein "Tierhaus". Weitere 1930 geplante Erweiterungsbauten U. a. für eine neve Schmiede, für die "Geburtshilfliche und Rinderklinik" sowie für die "Medizinisch-forensische Klinik" kamen vor der Machtergreifung der Nationalsozialisten nicht mehr zur Ausführung. Im "Dritten Reich" wurde 1934 nur noch der Neubau der Schmiede fertiggestellt (Dahmen und Wagener 1930, Fröhner 1950, Ruthe und Drawer 1957).

Von den während der Weimarer Republik berufenen Ordina- rien hatte Wilhelm Noeller (1890-1964) bereits während des I. Weltkrieges allgemeine Anerkennung gefunden, als er die Schwefeldioxidbehandlung der Pferderäude in speziell dazu konstruierten Begasungszellen einführte. Er wurde dafür 1921 als Erster mit der "Dammann-Medaille" der Tierärztlichen Hochschule Hannover ausgezeichnet. Noeller hatte von 1910 bis 1914 in Berlin und München Veterinärmedizin studiert und befasste sich bereits als Student mit parasitologischen Fragen. Nach Kriegsende war er von 1919 bis 1921 am Institut für Tropenkrankheiten im Hamburg tätig und 1920/21 auch Privatdozent für Protozoologie an der Medizinischen Fakultät der Hamburger Universität. 1921 übernahm er die Nachfolge von Wilhelm Schütz als Direktor des Instituts für Veterinärpathologie der Berliner Tierärztlichen Hochschule. 1926 gab er den Lehrstuhl für Veterinärpathologie an Johannes Dobberstein (1895-1965) ab, um sich von da an ganz der Parasitologie zu widmen. Er unterhielt zahlreiche wissenschaftliche Kontakte mit dem Ausland, insbesondere zu dem weltberühmten russischen Helmithologen Konstantin Ivanovic Skrjabin (1878-1972). Als Sozialdemokrat geriet er aber in Konflikt mit den konservativen Kräften der Tierärztlichen Hochschule. Noeller wurde nach dem Machtantritt der Nationalsozialisten im April 1933 aus dem Hochschuldienst entlassen (Meissner 1990).

Der 1923 als Ordinarius für Tierzucht berufene Valentin Stang (1876-1944) wurde vor allem durch die gemeinsam mit dem Wiener Kliniker David Wirth (1885-1971) herausgebrachte Enzyklopädie "Tierheilkunde und Tierzucht" in 10 Bänden (1926-1932) mit einem 11. Ergänzungsband (1937) bekannt, die auch zahlreiche Beiträge über Pferdezucht und Pferdeheilkunde enthält.

Die Vorherrschaft der Nationalsozialisten an der Berliner Tierärztlichen Hochschule begann schon vor Hitlers Machtantritt mit der 1929 erfolgten Gründung einer Sektion des "Nationalsozialistischen Studentenbundes" (Hass 1989, Deutrich 1990) und mit dem 1932 erfolgten Eintritt von Wilhelm Krüger (1898-1977) in die NSDAP. Krüger wurde 1933 zum Rektor der Tierärztlichen Hochschule ernannt und präsentierte sich mit der Amtskette in Naziuniform. Nach der Eingliederung der Tierärztlichen und Landwirtschaftlichen Hochschulen als Landwirtschaftlich-Tierärztliche Fakultät in die Berliner Universität wurde Krüger im April 1935 in SA-Uniform auch als Rektor der Universität inthronisiert (Anonym 1933, 1935, Klee 2003). 1937 wurde die Veterinärmedizinische Fakultät selbständig und Krüger befasste sich dann bis 1945 als Veterinäranatom vorwiegend mit der Pferdeanatomie: Neben Studien über den Bewegungsablauf bei Pferden (Krüger 1937, 1938,1939 u. 1939 a) erschien u. a. 1939 auch sein Buch "Unser Pferd und seine Vorfahren".

Zum Ende des "Ill. Reiches" verfügte die Veterinärmedizinische Fakultät über 14 Ordinariate (das 14. Ordinariat wurde nach der Trennung von der Kleintierklinik 1937 für die Pharmakologie und Toxikologie eingerichtet), 4 Honorarprofessuren, 3 außerordentliche Professuren, 4 Dozenturen und 7 Lehrbeauftragte (Beutner 1940). Von den 14 ordinierten Professoren waren 11 Mitglieder der NSDAP, 5 waren auch in der SA und weitere 2 waren Mitglieder der SS. Zu den Honorarprofessoren gehörte neben dem Veterinärinspekteur beim Oberkommando des Heeres auch der Reichstierärzte- und höhere SS-Führer Friedrich Weber (1892-1955) (Anonym 


\section{Pferdeheilkunde \\ Behandlungsvertrag}

Regressanspruch in der Pferdepraxis? Wurde umfassend und angemessen aufgeklärt? Ist die Behandlung vollständig dokumentiert?

Der Pferdeheilkunde-Behandlungsvertrag hilft, den Umfang der Aufklärung zu belegen und er hält zu regelmäßiger Dokumentation an.

Treffen Sie für besonderes langwierige, risikobehaftete und aufwändige Behandlungen, für alle operativen Eingriffe und Narkosen eine schriftliche Vereinbarung.

Schützen Sie sich bestmöglich gegen Ansprüche von Seiten der Pferdebesitzer. 
1939 a, Klee 2003). Auch die übrigen Angehörigen des Lehrkörpers waren mehrheitlich Mitglieder der NSDAP oder höhere Veterinäroffiziere, die beim Eintritt in die Wehrmacht ihre Mitgliedschaft in der NSDAP aufgeben mussten (Schimanski 2004).

Mit Beginn der Naziherrschaft wurden wie an allen höheren Bildungsanstalten Juden, Marxisten und andere nicht national gesinnte Abiturienten nicht mehr zum Studium zugelassen. Jüdischen Tierärzten wurden auch in Berlin die Doktortitel aberkannt. Ihnen wurde 1939 auch noch die Approbation entzogen und "die Ausübung der ... Tierheilkunde überhaupt untersagt" (Anonym 1939, Möllers 2002).

Die enge Verquickung der Veterinärmedizinischen Fakultät mit der Militärveterinärwesen lebte während der Naziherrschaft nach Wiedereinführung der allgemeinen Wehrpflicht im Jahre 1935 wieder auf. Die Ausbildung der Studenten wurde jetzt auch auf die Bedürfnisse der Wehrmacht und der späteren Waffen-SS ausgerichtet (Heeresveterinärwesen, Gasschutz, Luftschutzveterinärdienst, Huf- und Beschlagskunde, Staatsveterinärkunde im Geiste des Nationalsozialismus). 1935/36 waren insgesamt 347 männliche und 12 weibliche Studierende in der Abteilung Tierheilkunde der Landwirtschaftlich-Tierärztlichen Fakultät immatrikuliert; das Kontingent für das Tierarztstudium wurde 1936 erhöht; genave Zahlen wurden aber nicht bekanntgegeben (Anonym 1935 a, 1936, Beutner 1940, Ohmke 1940).

Die "Alte Zootomie" erhielt 1936 einen weiteren Anbau, das sog. Schlachthaus für Demonstrationen auf dem Gebiet der Fleischbeschau/-untersuchung. Die Lehre und Forschung stand hier jetzt „im Dienst der Volkshygiene" (Anonym 1937, Lerche 1933) und während des II. Weltkrieges wurden in der "Alten Zootomie" vor allem Fragen zur Konservierung von Fleisch- und Wurstwaren für den Bedarf der Wehrmacht bearbeitet.

Das Ende des III. Reiches brachte auch für die Veterinärmedizinische Fakultät Zerstörungen der Gebäudesubstanz, Personenverluste und geistige Verwirrung. Die "Alte Zootomie" wurde durch zwei Bombentreffer stark in Mitleidenschaft gezogen, blieb aber zum Glück als Ganzes in ihrer ursprünglichen äußeren Form mit Einschluss des "Anatomischen Theaters" erhalten.

\section{Vom Neuanfang nach 1945 bis zur Wende 1990}

Die Entwicklung von Lehre und Forschung nach dem Ende des II. Weltkrieges wurde anlässlich des 200jährigen Jubiläums der Veterinärmedizinischen Fakultät in zwei Festschriften erschöpfend dargestellt. Hier bleibt nur zu erwähnen, dass sich von 14 Ordinarien ab 1946 nur 4 zum Wiederaufbau einfanden. Der Tierseuchentherapeut und der Pharmakologe begingen 1945 Selbstmord, der Chemiker kam bei den Kämpfen in Berlin um. Der Physiologe verstarb in sowjetischer Kriegsgefangenschaft. Der Tierzüchter verstarb bereits 1944, so dass der Lehrstuhl beim Neubeginn zunächst vakant war. 5 ordinierte Professoren, die Mitglieder der NSDAP (2 auch in der SS und 2 in der SA) waren, kehrten nicht an ihren Arbeitsplatz zurück. Sie fanden später in der Bundesrepublik oder in Westberlin eine Wiederverwendung als Hochschullehrer bzw. andere Arbeitsmöglichkeiten (Fröhner 1950, Schröder 1965, Schimanski 1999, 2004).

Eines der ersten Gebäude, das nach 1945 wieder für die Ausbildung von Tierärzten zur Verfügung stand, war die "Alte Zootomie". Neben der Lebensmittelhygiene fanden hier jetz† auch die Hygieniker, deren Lehr- und Forschungsstätte durch Bombeneinwirkung während des Krieges völlig ausgebrannt war, vorübergehend Unterkunft. Auch die Tierseuchentherapie, deren Leitung Martin Lerche (1892-1980) vorübergehend übernommen hatte, war hier untergebracht (Nusshag 1959/60).

Für die Pferdeheilkunde hatte die "Alte Zootomie" allerdings schon lange ihre Bedeutung verloren. Obwohl durch die zunehmende Motorisierung das Pferd aus Landwirtschaft und Verkehr mehr und mehr verdrängt wurde, wurde dennoch die Pferdeheilkunde weiter entwickelt. 1949 entstand das "Institut für Veterinär-Orthopädie", an dem neben Lehre und Forschung für die Veterinärmedizin auch Hufbeschlagschmiede ausgebildet wurden (Ruthe und Drawer 1957). Dieses Institut wurde später in den Verband der Chirurgischen Tierklinik aufgenommen.

1959 wurde die Rennbahnklinik in Hoppegarten an die Chirurgische Tierklinik angegliedert und nach modernen Gesichtspunkten für die stationäre Behandlung von Galoppund Traberrennpferden ausgebaut. Die Chirurgische Tierklinik übernahm auch die Betreuung von Trabergestüten im Umkreis von Berlin und der Chef der Hoppegartener Klinik betreute die Pferdeequipen der DDR bei nationalen und internationalen Turnieren. An der Chirurgischen Tierklinik der Humboldt-Universität wurden auch Pferdepatienten aus Westberlin und aus der Bundesrepublik stationär behandelt (Deutrich 1990).

Zu den Forschungsthemen, die an der Chirurgischen Tierklinik bearbeitet wurden, gehörten auch Aufgaben für die Pferdeheilkunde:Osteosynthese/Pferd, Schienbeinerkrankung/ Pferd und Kolikchirurgie beim Pferd. Zusammen mit dem Tierzüchter Ekkehard Wiesner (geb. 1924) gab der Direktor der Chirurgischen Tierklinik Olof Dietz (geb. 1927) auch ein Handbuch "Pferdekrankheiten" (1982) heraus, das 1984 als englischsprachige Ausgabe in Washington erschien. In der Medizinischen Klinik und in der Klinik für Geburtshilfe und Fortpflanzungsstörungen mit einer Besamungsstation, für die Anfang der 50er Neubaven errichtet wurden, trat die Pferdeheilkunde dagegen immer mehr in den Hintergrund.

Als Ganzes gesehen blieb die Veterinärmedizinische Fakultät der Humboldt-Universität von den politischen Auseinandersetzungen während der Existenz der DDR nicht unberührt. Anfang der 50er Jahre gingen 7 Professoren, darunter 4 nach 1946 neuberufene, zusammen mit mehreren Assistenten und etwa 200 Studenten zur Freien Universität nach Westberlin. An der Fakultät der Humboldt-Universität verblieben 6 ordinierte Professoren (Fortner 1951, 1952, Lerche 1965). Ein zweiter "Aderlass" erfolgte, als 1957 zwei weitere inzwischen neu berufene Institutsdirektoren zusammen mit mehreren Oberassistenten und Assistenten so wie einigen Studenten die Fakultät in Berlin-Mitte verließen. Nach dem Maverbau 1961 wechselte auch der in Westberlin wohnende Spezialist für Geflügelkrankheiten zur Freien Universität über. 
Den politischen Verhältnissen in der DDR Rechnung tragend, wurden auf die neu zu besetzenden Ordinariate vorwiegend Mitglieder der SED berufen. Zum Ende der DDR waren an der Veterinärmedizinischen Fakultät der Humboldt-Universität insgesamt 25 Professorinnen und Professoren tätig. 16 von ihnen waren Mitglieder der SED, ein Professor war CDU-Mitglied und 8 Professorinnen und Professoren gehörten keiner politischen Partei an.

\section{Nachbemerkung}

Die "Alte Zootomie" stand über 110 Jahre auch im Dienste der Pferdeheilkunde, denn hier wurde vorwiegend Pferdeanatomie betrieben. Zeitweise diente sie auch als Lehr- und Forschungsstätte für den Hufbeschlag, für die theoretische und praktische Ausbildung auf dem Gebiet der Pferdechirurgie und schließlich auch der Pferdepathologie. Im "Gerlachanbau" haben Wilhelm Schütz und sein Lehrer Rudolf Virchow die Pathologie des Pferderotzes erforscht und damit die Grundlagen für eine erfolgreiche Bekämpfung und Tilgung dieser Zoonose gelegt.

\section{Literatur}

Albers J. C. (1841): Geschichte der Königlichen Thierarzneischule zu Berlin, nebst Darstellung ihrer bisherigen Leistungen und gegenwärtigen Verfassung, Einladungsschrift zur Feier der Einweihung des neverbauten Thierarzneischulgebäudes und des fünfzigiährigen Bestehens der Anstalt am 2. Februar 1841, Berlin, gedruckt bei A. W. Schade, 41

Anonym (1933): Tierärztliche Hochschule zu Berlin, Tierärztl. Rundschau 39, 334-336, 569-570

Anonym (1935): Feierliche Rektoratsübergabe in der Berliner Universität, Tierärztl. Rundschau 41, 235-236

Anonym (1935 a): Landwirtschaftlich-Tierärztliche Fakultät der Friedrich-Wilhelms-Universität, Tierärzłl. Rundschau 41, 815

Anonym (1936): Erhöhung der Kontingentzahl der Studierenden der Landwirtschaftlich-Tierärztlichen Fakultät der Friedrich - Wilhelms Universität in Berlin, Tierärztl. Rundschau 42, 827

Anonym (1939): Ausschluss der Juden aus dem tierärztlichen Beruf, Tierärztl. Rundschau 45, 101

Anonym (1939 a): Die Von-Ostertag-Ehrung in Berlin, Tierärztl. Rundschau 45, 318

Bergmann V. und Wenzel M. (1990): Leben und Wirken von Wilhelm Schütz, erster Lehrstuhlinhaber für Veterinär-Pathologie an der tierärztlichen Ausbildungsstätte in Berlin, Mh. Vet.-Med. 45, 669-672

Beutner K. (1940): 150 Jahre Veterinärmedizinische Fakultät der Universität Berlin, Tierärztl. Rundschau 46, 245-254

Bongert (1931): Zum neunzigsten Geburtstage des Professors Dr. phil. Heinrich Möller, Berl. Tierärztl. Wochenschr. 47, 366-367

Dahmen H. und Wagener K. (1930): Die Tierärztliche Hochschule Berlin, Lindner-Verlag Küssnacht am Rigi, Düsseldorf

Deutrich V. (Hrsg.)(1990): Von der Königlichen Tierarzneischule zur Veterinärmedizinischen Fakultät der Humboldt - Universität zu Berlin 1790-1990, 200 Jahre veterinärmedizinische Ausbildung undForschung in Berlin, Quintessenz Verlag; München

Driesch A. von den und Peters J. (2003): Geschichte der Tiermedizin, 5000 Jahre Tierheilkunde, 2. Auflage, Schattaver GmbH Verlag für Medizin und Naturwissenschaften, Stuttgart, New York

Erdt W. E. A. (1862): Das Thierarzneiwesen im Preuss. Staate, Eine pragmatische Skizze zur Information für Landwirthe, Militairs, Thierärzte und Beamte, Berlin, Verlag Ernst Schotte

Farchmin G. (1965): Vúe de la Zootomie à Berlin, Wiss. Zeitschr. der Humboldt - Univ. zu Berlin, Sonderband,155 - 164

Fortner J. (1951): Zum Troste für unsere Ostkollegen, Berl. u. Münch. Tierärztl. Wochenschr. 64, 244
Fortner J. (1952): Niedriger hängen !, Berl. u. Münch. Tierärztl. Wochenschr. 65, 54

Froehner R. (1929): Zur Geschichte der Berliner Tierärztlichen Hochschule und ihres Erbavers, Tierärztl. Rundschau 35, 670-672

Fröhner R. (1950): Von der Tierarzneischule zur Veterinärmedizinischen Fakultät 1790 - 1950, Mh. Vet.-Med. 5, 107-126

Gerber T. (2004): Persönlichkeiten aus Land- und Forstwirtschaft, Gartenbau und Veterinärmedizin-Biographisches Lexikon, 2 Bände, NORA Verlagsgemeinschaft Dyck \& Westerheide, Berlin

Gurlt E. F. (1860): Hand-Atlas zu dem Handbuche der vergleichenden Anatomie der Haus-Säugethiere, Berlin, Verlag von August Hirschwald, XXII Tafeln mit Erläuterungen

Hass D. (Hrsg.) (1889): 200 Jahre Veterinärmedizin in Berlin, Wiss. Zeitschr. der Humboldt - Univ. zu Berlin, 38, 229-436

Hertwig (1836) Bericht über die Clinik in der Königlichen Thierarzneischule zu Berlin im Jahre 1835, Magazin f. d. ges. Thierheilkd., 2, 232-252, 326-351

Hertwig (1836 a): Nekrolog, Magazin f. d. ges. Thierheilkd. 2, 263264

Hertwig (1837): Kurzer Bericht über die Clinik in der Königlichen Thierarzneischule im Jahre 1836, Magazin f. d. ges. Thierheilkd. 3, 480-490

Hertwig (1840): Summarischer Bericht über die in dem Thierspital der Königl. Thierarzneischule zu Berlin in den Jahren von 1837 und 1838 verpflegten kranken Thiere, Magazin f. d. ges. Thierheilkd. 6, 129-142

Klee E. (2003): Das Personenlexikon zum Dritten Reich, Wer war was vor und nach 1945?, S. Fischer Verlag, Frankfurt am Main

Koch T. (1965): Zur Geschichte der Veterinärmedizinischen Fakultät der Humboldt - Universität zu Berlin, Wiss. Zeitschr. der Humboldt - Univ. zu Berlin, Sonderband, 9 - 52

Koch T. (1965 a): Geschichte des Instituts für Veterinär-Anatomie, Wiss. Zeitschr. der Humboldt-Univ. zu Berlin, Sonderband, 117-125

Krokotsch B. (1991): Tierhaltung und Veterinärmedizin im Berlin des 19. Und 20. Jahrhunderts, Eine Spurensicherung, Band 17 der Reihe Wissenschaft und Stadt, Publikationen der Freien Universität Berlin aus Anlass der 750-Jahr-Feier Berlins, Colloquium Verlag, Berlin

Krüger W. (1937): Ueber den Bewegungsablauf an dem oberen Teil der Vordergliedmasse des Pferdes im Schritt, Trab und Galopp, Tierärztl. Rundschau 43, 809-816, 825-827

Krüger W. (1938): Ueber den Bewegungsablauf an dem oberen Teil der Hintergliedmasse des Pferdes im Schritt, Trab und Galopp, Tierärztl. Rundschau 44, 549-557

Krüger W. (1939): Ueber die Arbeit der vier Gliedmassen des Pferdes beim Galopp, Tierärztl. Rundschau 45, 250-255

Krüger W. (1939 a): Bemerkungen zur Gliedmassenfolge beim Renngalopp und beim Sprung des Pferdes, Tierärztl. Rundschau 45, 287-289

Kühn E. (1994): Was wird aus Friedrichs Lieblingspferd, wenn der Professor geht, Die anatomische Sammlung des Humboldt-Veterinärs Tankred Koch hat die DDR überstanden, ist aber erneut in Gefahr, Tagesspiegel vom 21. 2. 1994;

Leisering A. G. T. und Mueller C. (1873): E. F. Gurlt's Handbuch der vergleichenden Anatomie der Haus-Säugethiere, neu bearbeitet, fünfte Auflage, Berlin, Verlag von August Hirschwald

Lerche M. (1933): Die Veterinärmedizin im Dienst der Volkshygiene, Tierärztl. Rundschau 39, 855-856

Lerche M. (1965): 175 Jahre tierärztliche Lehre und Forschung in Berlin, Festvortrag in "Zur 175. Wiederkehr des Gründungstages der Königlichen Tierarzneischule Berlin“, Hrsg: Veterinärmedizinische Fakultät der Freien Universität Berlin, Verlag Paul Parey, Berlin und Hamburg

Lotes-Köln (1925): Reinhold Schmaltz zum 65. Geburtstag, Berl. Tierärztl. Wochenschr. 41, 551-555

Lutz U. (2003): Carl Heinrich August Graf von Lindenau, 1789 1806 Obermarstallmeister, Begründer des Preussischen Gestütswesens, bis 1813 Chef der preussischen Haupt- und Landgestüte; Manuskript mit biographischen Notizen, 6 Seiten, teilweise in Tageszeitungen veröffentlicht;

Materna I. und Ribbe W. (Hrsg.)(1997): Geschichte in Daten Berlin, Koehler \& Amelang, München/Berlin 
Meissner C. (1990): Professor Dr. Wilhelm Nöller - dem Wegbereiter der Parasitologie in Deutschland zum 100. Geburtstag, Mh. Vet.-Med. 45, 673-675

Mielke H. (2005): Ein Jubiläumsgeburtstag steht bevor, Carl Heinrich August Graf von Lindenau ehemaliger Königlich-preussischer Oberstallmeister und Generaldirektor der Königlichen Thierarzneischule zu Berlin, wurde vor 250 Jahren im sächsischen Machern geboren, Manuskript, redaktionell gekürzt veröffentlicht in Dt. Tierärztebl. 53, 140-141

Möllers G. (2002): Jüdische Tierärzte im Deutschen Reich in der Zeit von 1918 bis 1945, Vet. med. Diss., Hannover

Müller (1890): Das anatomische Institut, in Schütz, W. (Hrsg.)(1890):

Die Thierärztliche Hochschule zu Berlin 1790 - 1890, Festschrift, Berlin, Verlag von August Hirschwald, 243

Müller E. Hrsg. (1990): Gelegentliche Gedanken über Universitäten von J. J. Engel, J. B. Erhard, F. A. Wolf, G. Fichte, F. D. E. Schleiermacher, K. F. Savigny, W. v. Humboldt, G. F. W. Hegel, Reclam Verlag Leipzig, 49 - 50, 282, 297-298, 301

Niepage H. (1988): J. G. Naumann (1754 - 1836), Königliche Thierarzneischule zu Berlin, Specielle Pathologie und Therapie nach der Skripte des Eleven E. A. Schrader entziffert und erläutert, Selbstverlag Berlin

Nusshag W. (1959/60): Zur Geschichte der Veterinärmedizinischen Fakultät und ihrer Institute, Wiss. Zeitschr. der Humboldt-Univ. zu Berlin, Beiheft zum Jubiläumsjahrgang (IX) 1959/69, 215-228

Ohmke (1940): Die Beziehungen zwischen der Berliner veterinärmedizinischen Fakultät und dem Heeres-Veterinärwesen, Berl. u. Münch. Tierärztl. Wochenschr. 56, 315-320

Pusch (1909): Ein Ausflug nach österreich - ungarischen Gestüten, Dtsch. Tierärztl. Wschr. 17, 709-714

Rieck W. (1928): Zur ältesten Geschichte der Tierärztlichen Hochschule Berlin, Vet.hist. Jahrb. IV, 78-144

Rieck W. (1934): Taten und Pläne des Professors Sick, Berl. Tierärztl. Wochenschr. , 90-92

Rieck W. (1934 a): Krankengeschichte des Maultiergestüts in Neustadt an der Dosse (1770-1776), Berl. Tierärztl. Wochenschr. 50, 622623

Rieck W. (1937): Krankengeschichte des Friedrich-Wilhelm-Gestüts in Neustadt an der Dosse (1791), Berl. Tierärztl. Wochenschr. 53, 545548

Rieck W. (1943); J. G. Naumanns Bericht über Druse im FriedrichWilhelm-Gestüt 1820, Tierärztl. Rundschau 49, $42-43$

Rieck W. (1943 a): J. G. Naumanns Lehriahre, Berl. u. Münch. Tierärztl. Wochenschr. u. Wiener Tierärztl. Monatsschr 59, 308-309

Ruthe H. und Drawer K. (1957): Die Entwicklung der Lehrschmiede Berlin, Mh. Vet.-Med. 12, 436-441

Schimanski M. (1999): Die Entnazifizierung an den tierärztlichen Bildungsstätten in Deutschland, 7. Tagung der Fachgruppe "Geschichte der Veterinärmedizin" der DVG 12.-13. November 1999 in Hannover, DVG-Tagungsband, Giessen, 34-49

Schimanski M. (2004): NSDAP-Mitgliedschaften des Lehrkörpers der Berliner Fakultät von 1940, persönl. Mitteilung

Schmaltz (1912): Das erste Vierteljahrhundert der Berliner Tierärztlichen Hochschule, Berl. Tierärztl. Wochenschr. 28, 438-439

Schmaltz R. (1936): Entwicklungsgeschichte des tierärztlichen Berufes und Standes in Deutschland, Berlin, Verlagsbuchhandlung von Richard Schoetz

Schneck P. (1997): Geschichte der Medizin, systematisch, UNI MED Verlag AG, Bremen und Lorch/Württemberg

Schrader G. W. und Hering E. (1863): Biographisch-literarisches Lexicon der Thierärzte aller Zeiten und Länder, sowie der Naturforscher, Aerzte, Landwirthe, Stallmeister u. s. w., welche sich um die Thierheilkunde verdient gemacht haben, Stuttgart, Verlag von Ebner \& Seubert

Schröder F. (1990): In der Fakultät der Veterinärmedizin entdeckt: "Condé", das letzte Leibpferd Friedrich des Großen, ND vom 25. 7. 1990
Schröder K. Hrsg.(1965): Veterinärmedizin in Berlin 1790-1965; Geschichte, Lehre und Forschung heute, Der Tierarzt im Sozialismus, Wiss. Zeitschr. der Humboldt-Univ. zu Berlin, Sonderband, Schütz W. Hrsg.(1890): Die Thierärztliche Hochschule zu Berlin 1790-1890, Festschrift, Berlin, Verlag von August Hirschwald,

Schwarz A. (1967): Tierseuchenbekämpfung anno 1797, Tierärztl. Umschau 22, 530-533

Skriabin K. I. Hauptredakteur (1969): Gel'man Christofor Ivanovic (Kurzbiographie) in: Veterinär-Enzyklopädie, Verlag "Sowjetische Enzyklopädie", Moskau (russisch) Band 2, 309

Skriabin K. I. Hauptredakteur (1972): Kal'ning, Otton Ivanovic (Kurzbiographie) in: Veterinär-Enzyklopädie, Verlag "Sowjetische Enzyklopädie", Moskau (russisch) Band 3, 267

Skriabin K. I. Hauptredakteur (1975): Skriabin, Konstantin Ivanovic (Kurzbiographie) in: Veterinär-Enzyklopädie, Verlag "Sowjetische Enzyklopädie", Moskau (russisch) Band 5, 730-732

Stang W. und Wirth D. Hrsg. (1927): Gestütswesen. in: Tierheilkunde und Tierzucht, Eine Enzyklopödie der praktischen Nutztierkunde, Urban \& Schwarzenberg, Berlin u. Wien, Band 4, 639-641

Stang W. und Wirth D. Hrsg. (1928): Gurlt u. Hertwig (Kurzbiographien) in: Tierheilkunde und Tierzucht, Eine Enzyklopädie der praktischen Nutztierkunde, Urban \& Schwarzenberg, Berlin u. Wien, Band 5, 25 und 242

Stang W. und Wirth D. Hrsg. (1930): Naumann (Kurzbiographie) in: Tierheilkunde und Tierzucht, Eine Enzyklopädie der praktischen Nutztierkunde, Urban \& Schwarzenberg, Berlin u. Wien, Band 7, 367

Stang W. und Wirth D. Hrsg. (1931): Sick (Kurzbiographie) in: Tierheilkunde und Tierzucht, Eine Enzyklopädie der praktischen Nutztierkunde, Urban \& Schwarzenberg, Berlin u. Wien, Band 9, 410

Toellner R. Hrsg. (2000): Illustrierte Geschichte der Medizin, Band 6, S. 3263, Lizenzausgabe für Weltbild Verlag $\mathrm{GmbH}$, Augsburg

Wernicke R. K. H. (1996): Veterinärmedizin und Verkehr, Ein historischer Überblick, Vortragsmanuskript, Kongress der "World Association for the History of Veterinary Medicin" Oktober 1996 in Wien; gekürzt veröffentlicht in "Neterinärwesen und Tiermedizin im Sozialismus", Band 2, Hrsg. von Martin Fritz Brumme und Hartwig Prange, 133-158

Wernicke R. K. H. (1997): Die Formierung des Veterinärwesens im besetzten Polen 1939-1945, 5. Tagung der Fachgruppe "Geschichte der Veterinärmedizin": Veterinärmedizin im Dritten Reich, Tagungsbericht, Verlag der DVG, Giessen, 214-261

Wernicke R. K. H. (2000): Die Denkmale für die Tierärzte Andreas Christian Gerlach, Wilhelm Dieckerhoff und Wilhelm Schütz in Berlin, 8. Tagung der Fachgruppe "Geschichte der Veterinärmedizin": Tiermedizin im Spiegel der Kunst, Literatur und Musik, Tagungsbericht, Verlag der DVG, Giessen, 40-63

Wernicke R. K. H. (2000 a): Medizin und Veterinärmedizin, Gemeinsamkeiten und Probleme, Eine historische und aktuelle Reflektion aus der Sicht eines Veterinärmediziners, Veröff. Med. Ges.. 6, 1-125

Wernicke R. K. H. (2003): Die Scholaren der Königlichen Tierarzneischule Berlin (1790-1800), 11. Arbeitstagung der Fachgruppe "Geschichte": Student(in) der Tiermedizin-Einst und Jetzt, 27. Juni 2003 Tierärztliche Hochschule Hannover-225 Jahre, Tagungsbericht, Verlag: DVG Service GmbH, Giessen, 62 - 78

Wernicke R. K. H. (2004): Anmerkungen zur Geschichte der "Alten Zootomie", Selbstverlag Berlin

Dr. Rudolf Wernicke

Schönefelder Chaussee 177

12524 Berlin Waldesruh

wernickemoritz@aol.com

\section{Pferdeheilkunde Behandlungsvertrag}

